\title{
Symplectic harmonicity and generalized coeffective cohomologies
}

\author{
Luis Ugarte ${ }^{1} \cdot$ Raquel Villacampa ${ }^{2}$
}

Received: 20 July 2018 / Accepted: 17 December 2018 / Published online: 7 January 2019 (c) Fondazione Annali di Matematica Pura ed Applicata and Springer-Verlag GmbH Germany, part of Springer Nature 2019

\begin{abstract}
Relations between the symplectically harmonic cohomology and the coeffective cohomology of a symplectic manifold are obtained. This is achieved through a generalization of the latter, which in addition allows us to provide a coeffective version of the filtered cohomologies introduced by Tsai, Tseng and Yau. We construct closed (simply connected) manifolds endowed with a family of symplectic forms $\omega_{t}$ such that the dimensions of these symplectic cohomology groups vary with respect to $t$. A complete study of these cohomologies is given for 6-dimensional symplectic nilmanifolds, and concrete examples with special cohomological properties are obtained on an 8-dimensional solvmanifold and on 2-step nilmanifolds in higher dimensions.
\end{abstract}

Keywords Symplectic Hodge theory · Coeffective cohomology · Filtered and primitive cohomologies · Lefschetz map

Mathematics Subject Classification 53D05 · 53D35 · 57R17

\section{Introduction}

Let $\left(M^{2 n}, \omega\right)$ be a symplectic manifold. The notion of symplectically harmonic form was introduced by Brylinski [7] as a closed form $\alpha$ such that its symplectic star is also closed, i.e. $d \alpha=0=d * \alpha$. Mathieu [20] proved (see also [29] for a different proof) that every de Rham cohomology class has a symplectically harmonic representative if and only if $\left(M^{2 n}, \omega\right)$ satisfies the Hard Lefschetz Condition (HLC for short), i.e., the homomorphisms $L^{k}: H^{n-k}(M) \longrightarrow H^{n+k}(M)$ are surjective for every $1 \leq k \leq n$. Here $H^{q}(M)$ denotes the

$凶$ Raquel Villacampa

raquelvg@unizar.es

Luis Ugarte

ugarte@unizar.es

1 Departamento de Matemáticas, IUMA, Universidad de Zaragoza, Campus Plaza San Francisco, 50009 Zaragoza, Spain

2 Centro Universitario de la Defensa Zaragoza, IUMA, Universidad de Zaragoza, Academia General Militar, Crta. de Huesca s/n, 50090 Zaragoza, Spain 
$q$ th de Rham cohomology group of $M$ and $L^{k}$ is the homomorphism given by the cup product with the class $\left[\omega^{k}\right] \in H^{2 k}(M)$. Since there exist many symplectic manifolds which do not satisfy the HLC, one has that the quotient $H_{\mathrm{hr}}^{q}(M)=\Omega_{\mathrm{hr}}^{q}(M) /\left(\Omega_{\mathrm{hr}}^{q}(M) \cap \operatorname{im} d\right), \Omega_{\mathrm{hr}}^{q}(M)$ being the space of symplectically harmonic $q$-forms, counts the de Rham cohomology classes in $H^{q}(M)$ containing harmonic representative.

Additional symplectic invariants of cohomological type were introduced by Bouché [6] as follows. A differential form $\alpha$ is called coeffective if it annihilates $\omega$, i.e. $\alpha \wedge \omega=0$. The space of coeffective forms with the (restriction of the) exterior derivative provides a subcomplex of the de Rham complex that is elliptic in any degree $q \neq n$. It turns out [6] that for compact Kähler manifolds $\left(M^{2 n}, \omega\right)$ and for every $q \geq n+1$, the $q$ th coeffective cohomology group, that we will denote here by $H_{(1)}^{q}(M)$, is isomorphic to the [ $\left.\omega\right]$-truncated qth de Rham group. However, this is no longer true for arbitrary compact symplectic manifolds [11]. On the other hand, Tseng and Yau $[26,27]$ have developed a symplectic Hodge theory by considering various cohomologies where the primitive cohomologies $P H_{d+d^{\Lambda}}(M), P H_{d d^{\Lambda}}(M), P H_{\partial_{+}}(M)$ and $P H_{\partial_{-}}(M)$ play a central role. Recently, Eastwood [10] has introduced an extension of the coeffective complex which is elliptic in any degree and such that the corresponding cohomology groups are isomorphic to the primitive cohomology groups.

The symplectically harmonic cohomology and the coeffective cohomology, to our knowledge, have been studied separately in the literature. Our first goal in this paper is to obtain some relations between both cohomologies by considering a natural generalization of the coeffective cohomology, which in addition will allow us to provide a coeffective version of the filtered cohomologies. The latter have recently been introduced by Tsai et al. [25], and extend the primitive cohomologies [26,27].

Another aspect in the study of the symplectic harmonicity is the notion of flexibility, motivated by the following question, which seems to be related to some problems of group-theoretical hydrodynamics [1], posed by Khesin and McDuff (see [29]): which closed manifolds $M$ possess a continuous family $\omega_{t}$ of symplectic forms such that the dimension of $H_{\mathrm{hr}}^{q}\left(M, \omega_{t}\right)$ varies with respect to $t$ ? Yan [29] proved the existence of a 4-dimensional flexible manifold, whereas in [16] several 6-dimensional nilmanifolds satisfying such property were found. Recently, Cho [8] has proved the existence of simply connected flexible examples of dimension six. Our second goal in this paper is to relate the harmonic flexibility to corresponding notions of flexibility for the generalized coeffective and filtered cohomologies, as well as to construct closed manifolds which are flexible with respect to these symplectic cohomologies.

In greater detail, the paper is structured as follows.

In Sect. 2 we introduce and study the generalized coeffective cohomologies of a symplectic manifold $\left(M^{2 n}, \omega\right)$. For each integer $k, 1 \leq k \leq n$, we consider the complex of $k$-coeffective differential forms as the subcomplex of de Rham one constituted by all the forms that annihilate $\omega^{k}$. The associated cohomology groups are denoted by $H_{(k)}^{q}(M)$. This complex is elliptic in any degree $q \neq n-k+1$, however one can define in a natural way a quotient $\hat{H}^{n-k+1}(M)$ of $H_{(k)}^{n-k+1}(M)$ which shares the same properties as the cohomology groups $H_{(k)}^{q}(M), q \geq n-k+2$ (see Propositions 2.5 and 2.7). The spaces $\hat{H}^{1}(M), \ldots, \hat{H}^{n}(M)$ play an important role in this paper since they will allow us to relate the different symplectic cohomologies involved. We will refer to the collection

$$
\hat{H}^{n-k+1}(M), H_{(k)}^{n-k+2}(M), \ldots, H_{(k)}^{2 n}(M), \quad 1 \leq k \leq n,
$$

as the generalized coeffective cohomology groups of the symplectic manifold $\left(M^{2 n}, \omega\right)$. It turns out that these spaces are symplectic invariants that only depend on the de Rham 
class $\left[\omega^{k}\right] \in H^{2 k}(M)$ (see Remark 2.10 and Lemma 2.11). When $M$ is of finite type, in Proposition 2.9 we prove that, for each $1 \leq k \leq n$, the alternating sum $\chi^{(k)}(M)$ of the dimensions of the generalized $k$-coeffective cohomology groups only depends on the topology of the manifold $M$.

Eastwood [10] has introduced an elliptic extension of the usual coeffective complex (i.e. $k=1$ ) such that the corresponding cohomology groups are isomorphic to primitive cohomology groups defined by Tseng and Yau [26,27]. In Sect. 3, for any $1 \leq k \leq n$, we consider an extension of the $k$-coeffective complex, which is also elliptic, whose cohomology groups $\check{H}_{(k)}^{q}(M)(0 \leq q \leq 2 n+2 k-1)$ are isomorphic to the filtered cohomology groups introduced by Tsai et al. [25] (see Remark 3.7 for details); in particular,

$$
\check{H}_{(1)}^{q}(M) \cong P H_{\partial_{+}}^{q}(M), \quad \check{H}_{(1)}^{2 n-q+1}(M) \cong H_{(1)}^{2 n-q}(M) \cong P H_{\partial_{-}}^{q}(M), \quad 0 \leq q \leq n-1,
$$

and

$$
\check{H}_{(k)}^{n+k-1}(M) \cong P H_{d d^{\Lambda}}^{n-k+1}(M), \quad \check{H}_{(k)}^{n+k}(M) \cong P H_{d+d^{\Lambda}}^{n-k+1}(M), \quad 1 \leq k \leq n .
$$

In Proposition 3.1, we show that these extended cohomologies also satisfy the main properties of the generalized coeffective cohomology groups. When $M$ is of finite type, we consider $\check{\chi}_{+}^{(k)}(M)$ as the alternating sum of the dimensions of the cohomology groups of the first half of the extended complex, and in Corollary 3.6 we prove the following characterization of the HLC: $\left(M^{2 n}, \omega\right)$ satisfies the HLC if and only if $\check{\chi}_{+}^{(k)}(M)=\chi^{(k)}(M)$ for every $1 \leq k \leq n$.

In Sect. 4 we obtain some relations of the generalized coeffective cohomologies (and therefore also of the filtered cohomologies) with the symplectically harmonic cohomology. Concretely, using the description of $H_{\mathrm{hr}}^{q}(M)$ obtained in $[16,28,29]$ we prove that the generalized coeffective cohomologies measure the differences between the harmonic cohomology groups in the following sense: if $\left(M^{2 n}, \omega\right)$ is a symplectic manifold of finite type, then

$$
\operatorname{dim} H_{\mathrm{hr}}^{n-k+1}(M)-\operatorname{dim} H_{\mathrm{hr}}^{n+k+1}(M)=\operatorname{dim} \hat{H}^{n-k+1}(M)
$$

for every $k=1, \ldots, n$ (see Theorem 4.4). As a consequence, we find the relation between the dimension of the primitive cohomology group $P H_{d+d^{\Lambda}}^{q}(M)$ and the harmonic cohomology for $q=1,2,3$.

We introduce in Sect. 5 the notion of generalized coeffective flexibility and filtered flexibility, as an analogous notion of the concept of harmonic flexibility. We say that a closed smooth manifold $M^{2 n}$ is c-flexible (resp. f-flexible or h-flexible) if $M$ possesses a continuous family of symplectic forms $\omega_{t}$ such that the dimension of some generalized coeffective (resp. filtered or symplectically harmonic) cohomology group varies with $t$. We prove in Theorem 5.3 that in four dimensions $M$ is never $c$-flexible, and that $M$ is $f$-flexible if and only if it is $h$-flexible. This result allows us to prove, for each $n \geq 2$, the existence of $2 n$-dimensional $f$-flexible closed manifolds having a continuous family of symplectic forms $\omega_{t}$ such that the dimension of the primitive cohomology group $P H_{d+d^{\Lambda}}^{2}\left(M, \omega_{t}\right)$ varies with respect to $t$ (see Theorem 5.6). In Theorem 5.7 we use a result in [8] to prove that, for every $n \geq 3$, there exists a $2 n$-dimensional simply connected closed manifold $M$ with a continuous family $\omega_{t}$ for which the dimensions of the primitive groups $P H_{d+d^{\Lambda}}^{3}\left(M, \omega_{t}\right)$ and $P H_{d d^{\Lambda}}^{3}\left(M, \omega_{t}\right)$ vary with $t$. In Theorem 5.8 and Proposition 5.11 we study flexibility in higher dimensions; in particular, it turns out that in dimension $2 n \geq 6$, if $M$ is $c$-flexible then $M$ is $f$-flexible or $h$-flexible. This shows that coeffective flexibility is a stronger condition than the other flexibilities.

All the cohomology groups can be computed explicitly for symplectic solvmanifolds satisfying the Mostow condition, in particular for any symplectic nilmanifold. In Sect. 6 
we consider the class of 6-dimensional nilmanifolds and compute the dimensions of all the cohomology groups for any symplectic form. This extends the previous study for the symplectically harmonic cohomology given in $[16,17]$. As a consequence, we identify all the 6-dimensional nilmanifolds which are $c$-flexible, $f$-flexible or $h$-flexible (see Table 1 ). A solvmanifold of dimension 8 that is $c$-flexible, $f$-flexible and $h$-flexible is described in Sect. 7. Section 8 is devoted to symplectic 2-step nilmanifolds, and based on results by Sakane and Yamada [23,28], we obtain examples of arbitrary high dimension which are $c$-flexible, $f$-flexible and $h$-flexible.

\section{Generalized coeffective cohomologies}

Let $\left(M^{2 n}, \omega\right)$ be a symplectic manifold of dimension $2 n$ and let $k$ be an integer such that $1 \leq k \leq n$. Next we introduce the notion of $k$-coeffective forms.

Definition 2.1 A $q$-form $\alpha$ on $M$ is said to be $k$-coeffective if $\alpha$ annihilates the form $\omega^{k}$, i.e. $\alpha \wedge \omega^{k}=0$. The space of $k$-coeffective forms of degree $q$ will be denoted by $\mathfrak{C}_{(k)}^{q}(M, \omega)$, or simply $\mathfrak{C}_{(k)}^{q}(M)$.

Remark 2.2 The above definition makes also sense in the "limit" case $k=n+1$ because $\omega^{n+1}=0$ and then $\mathfrak{C}_{(n+1)}^{*}(M)=\Omega^{*}(M)$. Also the case $k=0$ makes sense if we consider $\omega^{0}$ as the constant function 1 , i.e. $\mathfrak{C}_{(0)}^{*}(M)=\{0\}$. Thus, there exists the following strictly increasing sequence of differential ideals

$$
\{0\}=\mathfrak{C}_{(0)}^{*}(M) \subset \mathfrak{C}_{(1)}^{*}(M) \subset \cdots \subset \mathfrak{C}_{(n)}^{*}(M) \subset \mathfrak{C}_{(n+1)}^{*}(M)=\Omega^{*}(M) .
$$

Since for each $k$ the space $\mathfrak{C}_{(k)}^{*}(M)$ is closed by $d$, we can consider the $k$-coeffective complex

$$
\cdots \stackrel{d}{\longrightarrow} \mathfrak{C}_{(k)}^{q-1}(M) \stackrel{d}{\longrightarrow} \mathfrak{C}_{(k)}^{q}(M) \stackrel{d}{\longrightarrow} \mathfrak{C}_{(k)}^{q+1}(M) \stackrel{d}{\longrightarrow} \cdots,
$$

which is a subcomplex of the standard de Rham complex $\left(\Omega^{*}(M), d\right)$.

Definition 2.3 The qth $k$-coeffective cohomology group will be denoted by

$$
H_{(k)}^{q}(M)=\frac{\operatorname{ker}\left\{d: \mathfrak{C}_{(k)}^{q}(M) \longrightarrow \mathfrak{C}_{(k)}^{q+1}(M)\right\}}{\operatorname{im}\left\{d: \mathfrak{C}_{(k)}^{q-1}(M) \longrightarrow \mathfrak{C}_{(k)}^{q}(M)\right\}} .
$$

It is clear that the $k$-coeffective cohomology groups are invariant by symplectomorphism. Moreover, we will show below that, for each $k$, they are invariants of the de Rham class $\left[\omega^{k}\right] \in H^{2 k}(M)$.

Let $L_{\omega}^{k}: \Omega^{*}(M) \longrightarrow \Omega^{*}(M)$ be given by $L_{\omega}^{k}(\alpha)=\alpha \wedge \omega^{k}$. Since $\mathfrak{C}_{(k)}^{q}(M)=\operatorname{ker}\left\{L_{\omega}^{k}\right.$ : $\left.\Omega^{q}(M) \longrightarrow \Omega^{q+2 k}(M)\right\}$ and the map $L_{\omega}^{k}: \Omega^{q}(M) \longrightarrow \Omega^{q+2 k}(M)$ is injective for any $q \leq n-k$ and surjective for any $q \geq n-k$, one has that $H_{(k)}^{q}(M)=0$ for $q \leq n-k$ and $H_{(k)}^{q}(M) \cong H^{q}(M)$ for every $q \geq 2 n-2 k+2$.

The short exact sequence

$$
0 \longrightarrow \mathfrak{C}_{(k)}^{*}(M) \stackrel{i}{\longrightarrow} \Omega^{*}(M) \stackrel{L_{\omega}^{k}}{\longrightarrow} L_{\omega}^{k}\left(\Omega^{*}(M)\right) \longrightarrow 0,
$$


where $i$ denotes the inclusion, provides the following short exact sequence of complexes

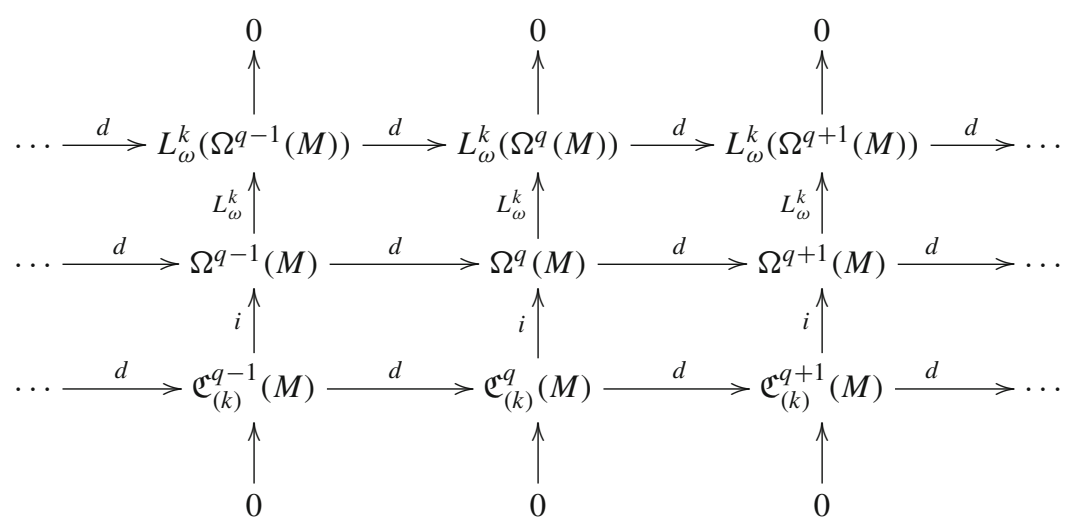

Now, since $L_{\omega}^{k}\left(\Omega^{q-2 k}(M)\right)=\Omega^{q}(M)$ for any $q \geq n+k$, we have that $H^{q}\left(L_{\omega}^{k}\left(\Omega^{*}(M)\right)\right)=$ $H^{q}(M)$ for $q \geq n+k+1$, and therefore the associated long exact sequence in cohomology is

$$
\begin{aligned}
& 0 \longrightarrow H^{n-k}(M) \stackrel{L^{k}}{\longrightarrow} H^{n+k}\left(L_{\omega}^{k}\left(\Omega^{*}(M)\right)\right) \stackrel{f_{n-k+1}}{\longrightarrow} H_{(k)}^{n-k+1}(M) \\
& \stackrel{H(i)}{\longrightarrow} H^{n-k+1}(M) \stackrel{L^{k}}{\longrightarrow} H^{n+k+1}(M) \stackrel{f_{n-k+2}}{\longrightarrow} H_{(k)}^{n-k+2}(M) \\
& \stackrel{H(i)}{\longrightarrow} H^{n-k+2}(M) \stackrel{L^{k}}{\longrightarrow} H^{n+k+2}(M) \stackrel{f_{n-k+3}}{\longrightarrow} H_{(k)}^{n-k+3}(M) \cdots,
\end{aligned}
$$

where $H(i)$ and $L^{k}$ are the homomorphisms in cohomology naturally induced by $i$ and $L_{\omega}^{k}$, respectively, and $f_{q}$ is the connecting homomorphism. Recall that $f_{q}$ is defined by $f_{q}([\alpha])=[d \beta]$, where $\beta \in \Omega^{q-1}(M)$ is any $(q-1)$-form satisfying $L_{\omega}^{k}(\beta)=\alpha$.

Definition 2.4 When the group $H_{(k)}^{q}(M)$ has finite dimension, we will denote it by $c_{q}^{(k)}(M)$ and we shall refer to it as the qth $k$-coeffective number of $\left(M^{2 n}, \omega\right)$.

Notice that $c_{q}^{(k)}(M)=0$ for any $q \leq n-k$, and $c_{q}^{(k)}(M)=b_{q}(M)$ for any $q \geq 2 n-2 k+2$.

In what follows, by a manifold of finite type we mean a manifold, not necessarily compact, such that its Betti numbers $b_{q}(M)=\operatorname{dim} H^{q}(M)$ are all finite.

Proposition 2.5 Let $\left(M^{2 n}, \omega\right)$ be a symplectic manifold of finite type and let $1 \leq k \leq n$. Then, for every $q \geq n-k+2$, the following properties hold:

(i) Finiteness and bounds for the coeffective numbers: the group $H_{(k)}^{q}(M)$ is finite dimensional and its dimension $c_{q}^{(k)}(M)$ satisfies the inequalities

$$
b_{q}(M)-b_{q+2 k}(M) \leq c_{q}^{(k)}(M) \leq b_{q}(M)+b_{q+2 k-1}(M) .
$$

(ii) Symplectic manifolds satisfying the HLC: if $\left(M^{2 n}, \omega\right)$ satisfies the HLC then the lower bound in (4) is attained, i.e.

$$
c_{q}^{(k)}(M)=b_{q}(M)-b_{q+2 k}(M) .
$$


(iii) Exact symplectic manifolds: if $\omega$ is exact, then the upper bound in (4) is attained, i.e.

$$
c_{q}^{(k)}(M)=b_{q}(M)+b_{q+2 k-1}(M) .
$$

(iv) Poincaré lemma: if $U$ is the open unit disk in $\mathbb{R}^{2 n}$ with the standard symplectic form $\omega=\sum_{i=1}^{n} d x^{i} \wedge d x^{n+i}$, then $c_{q}^{(k)}(U)=0$.

Proof From the long exact sequence (3), one has for any $q \geq n-k+2$ the five-term exact sequence

$$
0 \longrightarrow \operatorname{im} f_{q} \hookrightarrow H_{(k)}^{q}(M) \stackrel{H(i)}{\longrightarrow} H^{q}(M) \stackrel{L^{k}}{\longrightarrow} H^{q+2 k}(M) \stackrel{f_{q+1}}{\longrightarrow} \operatorname{im} f_{q+1} \longrightarrow 0 .
$$

If the manifold is of finite type, then it is clear that $H_{(k)}^{q}(M)$ has finite dimension for any $q \geq n-k+2$. Moreover, taking dimensions in (5)

$$
c_{q}^{(k)}(M)=\operatorname{dim}\left(\operatorname{im} f_{q}\right)+b_{q}(M)-b_{q+2 k}(M)+\operatorname{dim}\left(\operatorname{im} f_{q+1}\right),
$$

which implies the inequalities (4). This completes the proof of (i).

Property (ii) is a direct consequence of (5) taking into account that HLC implies that $L^{k}: H^{q-1}(M) \longrightarrow H^{q+2 k-1}(M)$ are surjective and then the connecting homomorphisms $f_{q}$ vanish for every $q \geq n-k+2$.

Property (iii) is a consequence of (5) since $L^{k}: H^{q-1}(M) \longrightarrow H^{q+2 k-1}(M)$ are identically zero because $\omega$ is exact, and then the connecting homomorphisms $f_{q}$ are injective for every $q \geq n-k+2$.

Finally (iv) is a direct consequence of (iii) since $\omega$ is exact on $U$.

Notice that for $k=1$ the previous proposition was proved by Fernández, Ibáñez and de León [12]. It is easy to check (see [6] for $k=1$ ) that, for each $1 \leq k \leq n$, the $k$-coeffective complex (2) is elliptic in any degree $q \neq n-k+1$. The coeffective group $H_{(k)}^{n-k+1}(M)$ can be infinite dimensional; however, in view of the sequence (3), there is a natural quotient of this coeffective group by considering the (in general also infinite dimensional) space $H^{n+k}\left(L_{\omega}^{k}\left(\Omega^{*}(M)\right)\right)$. We will see below that such quotient has finite dimension on symplectic manifolds of finite type.

Definition 2.6 Let us consider the space

$$
\hat{H}^{n-k+1}(M):=\frac{\left\{\alpha \in \Omega^{n-k+1}(M) \mid d \alpha=0 \text { and } \alpha \wedge \omega^{k}=0\right\}}{\left\{d \beta \mid \beta \in \Omega^{n-k}(M) \text { and } d \beta \wedge \omega^{k}=0\right\}} .
$$

If its dimension is finite, then we will denote it by $\hat{c}_{n-k+1}(M)$.

Hence, we have an additional collection of $n$ symplectic invariants given by $\hat{H}^{n-k+1}(M)$ for $k=1, \ldots, n$, that is,

$$
\hat{H}^{n}(M), \hat{H}^{n-1}(M), \ldots, \hat{H}^{2}(M), \hat{H}^{1}(M) .
$$

From now on, we will refer to the collection (1) as the generalized coeffective cohomology groups of the symplectic manifold $\left(M^{2 n}, \omega\right)$.

From the long exact sequence (3), we get the following isomorphisms

$$
\hat{H}^{n-k+1}(M) \cong \operatorname{im} H(i) \cong H_{(k)}^{n-k+1}(M) / \operatorname{im} f_{n-k+1},
$$


where $\operatorname{im} f_{n-k+1} \cong \frac{H^{n+k}\left(L_{\omega}^{k}\left(\Omega^{*}(M)\right)\right)}{L^{k}\left(H^{n-k}(M)\right)}$. Hence, we can consider the short exact sequence

$$
0 \longrightarrow \hat{H}^{n-k+1}(M) \stackrel{\hat{\imath}}{\longrightarrow} H^{n-k+1}(M) \stackrel{L^{k}}{\longrightarrow} H^{n+k+1}(M) \stackrel{f_{n-k+2}}{\longrightarrow} \operatorname{im} f_{n-k+2} \longrightarrow 0,
$$

where $\hat{\imath}$ is the homomorphism naturally induced by $H(i)$. Since $\hat{\imath}$ is injective, it is clear that $\hat{H}^{n-k+1}(M)$ is finite-dimensional whenever $H^{n-k+1}(M)$ is, and in such case, we have

$$
\hat{c}_{n-k+1}(M)=b_{n-k+1}(M)-b_{n+k+1}(M)+\operatorname{dim}\left(\operatorname{im} f_{n-k+2}\right) .
$$

Therefore, the properties obtained in Proposition 2.5 extend to the space $\hat{H}^{n-k+1}(M)$ as follows:

Proposition 2.7 Let $\left(M^{2 n}, \omega\right)$ be a symplectic manifold of finite type and let $1 \leq k \leq n$. The following properties hold for $\hat{c}_{n-k+1}(M)$ :

(i) Finiteness and bounds for the coeffective number $\hat{c}_{n-k+1}(M)$ : the space $\hat{H}^{n-k+1}(M)$ is finite dimensional and its dimension satisfies the inequalities

$$
b_{n-k+1}(M)-b_{n+k+1}(M) \leq \hat{c}_{n-k+1}(M) \leq b_{n-k+1}(M) .
$$

(ii) Symplectic manifolds satisfying the HLC: if $\left(M^{2 n}, \omega\right)$ satisfies the HLC then the lower bound in (6) is attained, i.e.

$$
\hat{c}_{n-k+1}(M)=b_{n-k+1}(M)-b_{n+k+1}(M) .
$$

(iii) Exact symplectic manifolds: if $\omega$ is exact then the upper bound in (6) is attained, i.e.

$$
\hat{c}_{n-k+1}(M)=b_{n-k+1}(M) .
$$

(iv) Poincaré lemma: if $U$ is the open unit disk in $\mathbb{R}^{2 n}$ with the standard symplectic form $\omega=\sum_{i=1}^{n} d x^{i} \wedge d x^{n+i}$, then $\hat{c}_{n-k+1}(U)=0$.

Inspired by the definition of the Euler characteristic of a manifold, we define the following symplectic invariants:

Definition 2.8 Let $\left(M^{2 n}, \omega\right)$ be a symplectic manifold of finite type. For each $1 \leq k \leq n$, we define

$$
\chi^{(k)}(M)=(-1)^{n-k+1} \hat{c}_{n-k+1}(M)+\sum_{i=n-k+2}^{2 n}(-1)^{i} c_{i}^{(k)}(M)
$$

The next proposition shows that each $\chi^{(k)}(M)$ is actually a topological invariant of the manifold.

Proposition 2.9 Let $\left(M^{2 n}, \omega\right)$ be a symplectic manifold of finite type. For any $1 \leq k \leq n$,

$$
\chi^{(k)}(M)=\sum_{r=n-k+1}^{n+k}(-1)^{r} b_{r}(M) .
$$

Proof The long exact sequence (3) implies

$$
0=\hat{c}_{n-k+1}-b_{n-k+1}+b_{n+k+1}+\sum_{j=2}^{n+k}(-1)^{j-1}\left(c_{n-k+j}^{(k)}-b_{n-k+j}+b_{n+k+j}\right) .
$$


Writing this sum in terms of $\chi^{(k)}$ we get

$$
(-1)^{n-k+1} \chi^{(k)}+\sum_{j=1}^{n+k}(-1)^{j} b_{n-k+j}-\sum_{j=1}^{n+k}(-1)^{j} b_{n+k+j}=0 .
$$

Since $b_{i}=0$ for $i \geq 2 n+1$, the previous equality reduces to:

$$
\begin{aligned}
0 & =(-1)^{n-k+1} \chi^{(k)}+\sum_{j=1}^{n+k}(-1)^{j} b_{n-k+j}-\sum_{j=1}^{n-k}(-1)^{j} b_{n+k+j} \\
& =(-1)^{n-k+1} \chi^{(k)}+\sum_{r=n-k+1}^{2 n}(-1)^{r-n+k} b_{r}-\sum_{r=n+k+1}^{2 n}(-1)^{r-n-k} b_{r} .
\end{aligned}
$$

Equivalently,

$$
\chi^{(k)}=\sum_{r=n-k+1}^{2 n}(-1)^{r} b_{r}-\sum_{r=n+k+1}^{2 n}(-1)^{r} b_{r}=\sum_{r=n-k+1}^{n+k}(-1)^{r} b_{r} .
$$

Observe that the Euler characteristic of $M$ is recovered if we allow $k=n+1$ (see Remark 2.2).

Remark 2.10 It is clear from the long exact sequence (3) that, for each $k$, the generalized $k$-coeffective cohomology groups (1) are invariants of the de Rham cohomology class $\left[\omega^{k}\right]$ given by the cap product of $[\omega]$ by itself $k$ times. Even more, if $\left[\omega^{k}\right] \neq 0$ and we denote by $\left[\left[\omega^{k}\right]\right]$ the corresponding element in $\mathbb{P}\left(H^{2 k}(M)\right)$, then all the generalized $k$-coeffective groups are invariants of $\left[\left[\omega^{k}\right]\right]$. In conclusion, if $\left(M^{2 n}, \omega\right)$ is a symplectic manifold and $\omega^{k}$ are not exact, then the generalized coeffective cohomologies only depend on the element $[[\omega]] \in \mathbb{P}\left(H^{2}(M)\right)$.

From this remark it follows

Lemma 2.11 Let $F:(M, \omega) \longrightarrow\left(M^{\prime}, \omega^{\prime}\right)$ be a diffeomorphism such that $F^{*}\left[\omega^{\prime}\right]=\lambda[\omega]$ for some nonzero $\lambda \in \mathbb{R}$. Then, for any $1 \leq k \leq n, \hat{H}^{n-k+1}\left(M^{\prime}\right) \cong \hat{H}^{n-k+1}(M)$ and $H_{(k)}^{q}\left(M^{\prime}\right) \cong H_{(k)}^{q}(M)$ for every $q \geq n-k+2$.

Notice that it suffices to know the de Rham cohomology of $M$ together with the action of $L^{k}$ on it, in order to know the generalized $k$-coeffective cohomology. This can be applied in particular to solvmanifolds satisfying the Mostow condition [21], that is to say, to compact quotients $G / \Gamma$ of solvable Lie groups $G$ by a lattice $\Gamma$ satisfying that the algebraic closures $\mathcal{A}\left(\operatorname{Ad}_{G}(G)\right)$ and $\mathcal{A}\left(\operatorname{Ad}_{G}(\Gamma)\right)$ are equal. In fact, under this condition, one has that the natural map $\left(\bigwedge^{*} \mathfrak{g}^{*}, d\right) \hookrightarrow\left(\Omega^{*}(M), d\right)$ from the Chevalley-Eilenberg complex of the Lie algebra $\mathfrak{g}$ of $G$ to the de Rham complex of the solvmanifold $M=G / \Gamma$ is a quasi-isomorphism, i.e. $H^{q}(M) \cong H^{q}(\mathfrak{g})$ for any $0 \leq q \leq \operatorname{dim} M$. The following result is straightforward from the long exact sequence in cohomology:

Proposition 2.12 Let $(M=G / \Gamma, \omega)$ be a 2 -dimensional symplectic solvmanifold satisfying the Mostow condition. Let $\mathfrak{g}$ be the Lie algebra of $G$ and let $\omega^{\prime} \in \bigwedge^{2} \mathfrak{g}^{*}$ be a left-invariant symplectic form representing the de Rham class $[\lambda \omega] \in H^{2}(M)$ for some $\lambda \neq 0$. Then, for any $1 \leq k \leq n$, the inclusion $\wedge^{*} \mathfrak{g}^{*} \hookrightarrow \Omega^{*}(M)$ induces isomorphisms $\hat{H}^{n-k+1}(M, \omega) \cong \hat{H}^{n-k+1}\left(\mathfrak{g}, \omega^{\prime}\right)$ and $H_{(k)}^{q}(M, \omega) \cong H_{(k)}^{q}\left(\mathfrak{g}, \omega^{\prime}\right)$ for every $q \geq n-k+2$. 
In particular, the previous result holds for nilmanifolds [22] and in the completely solvable case [15], i.e. when the adjoint representation $\operatorname{ad}_{X}$ has only real eigenvalues for all $X \in \mathfrak{g}$.

Note that for the usual coeffective cohomology, i.e. $k=1$ and $q \geq n+1$, this result was proved in [11] (see also [12]).

Remark 2.13 For other results on the de Rham cohomology of compact solvmanifolds $G / \Gamma$, even in the case that the solvable Lie group $G$ and the lattice $\Gamma$ do not satisfy the Mostow condition, see [9,14]. Notice that for infra-solvmanifolds Baues proved in [4] an analogous result to Nomizu's theorem about the isomorphism of its cohomology and that of a certain complex of left-invariant forms, result that is used in [18] to study the 1-coeffective cohomology of certain symplectic aspherical manifolds.

Remark 2.14 For general symplectic manifolds (not necessarily of finite type), from the long exact sequence (3), one has the following isomorphism

$$
\frac{H_{(k)}^{q}(M)}{\operatorname{ker} H(i)} \cong \operatorname{ker}\left\{L^{k}: H^{q}(M) \longrightarrow H^{q+2 k}(M)\right\}
$$

for every $q \geq n-k+2$, where $\operatorname{ker} H(i)=\operatorname{im} f_{q} \cong \frac{H^{q+2 k-1}(M)}{L^{k}\left(H^{q-1}(M)\right)}$. In particular, if the HLC is satisfied then $H_{(k)}^{q}(M) \cong \operatorname{ker}\left\{L^{k}: H^{q}(M) \longrightarrow H^{q+2 k}(M)\right\}$. Since any compact Kähler manifold satisfies the HLC, we conclude that, for any $q \geq n-k+2$, the $k$-coeffective group $H_{(k)}^{q}(M)$ is isomorphic to the space of de Rham cohomology classes that annihilate the class $\left[\omega^{k}\right]$. For $k=1$ this result was proved by Bouché [6], where he refers to the latter groups as the truncated de Rham groups. In [11,18] the relation of the 1-coeffective cohomology with the truncated de Rham cohomology is also investigated.

\section{Extension of the generalized coeffective complexes}

In [10] Eastwood introduced an elliptic extension of the usual coeffective complex and showed that the corresponding cohomology groups are isomorphic to the primitive cohomology groups defined by Tseng and Yau [26,27]. In this section, for every $1 \leq k \leq n$, we consider an extension of the $k$-coeffective complex and study its relation to the filtered cohomologies of Tsai et al. [25].

Let us fix $k$ such that $1 \leq k \leq n$. For each $q$, let us consider the quotient space $\check{\Omega}_{(k)}^{q}(M)=$ $\frac{\Omega^{q}(M)}{L_{\omega}^{k}\left(\Omega^{q-2 k}(M)\right)}$. We denote by $\check{d}: \check{\Omega}_{(k)}^{q}(M) \longrightarrow \check{\Omega}_{(k)}^{q+1}(M)$ the natural map induced by the exterior differential, i.e. $\check{d}(\check{\alpha})=(d \alpha)^{\swarrow}=d \alpha+L_{\omega}^{k}\left(\Omega^{q-2 k+1}(M)\right)$, for any $\check{\alpha} \in \check{\Omega}_{(k)}^{q}(M)$. Then, we have the following complex

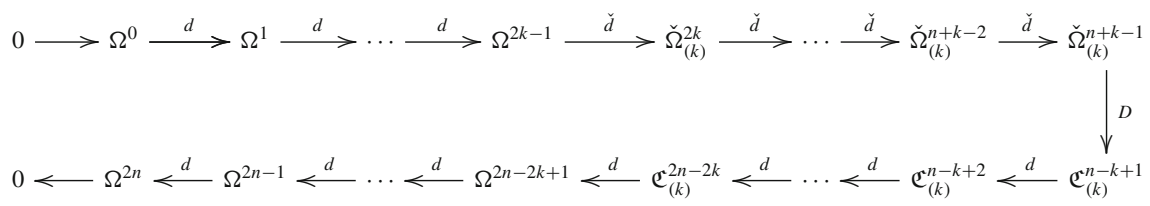

where $D$ is a second-order differential operator defined as $D(\check{\alpha})=d \gamma$, being $\gamma$ the unique $(n-k)$-form satisfying $d \alpha=L_{\omega}^{k}(\gamma)$. It can be checked that this complex is elliptic in any degree; however, we will not use this fact in what follows since the main properties of its cohomology groups will be derived from a long exact sequence as in Sect. 2. 
Let us denote by $\check{H}_{(k)}^{q}(M)$ the cohomology groups associated with the complex (7) for $0 \leq q \leq 2 n+2 k-1$. Notice that $\check{H}_{(k)}^{q}(M)=H^{q}(M)$ for any $q \leq 2 k-2$ and $\check{H}_{(k)}^{q}(M)=$ $H_{(k)}^{q-2 k+1}(M)$ for any $q \geq n+k+1$.

Now, the sequences of complexes

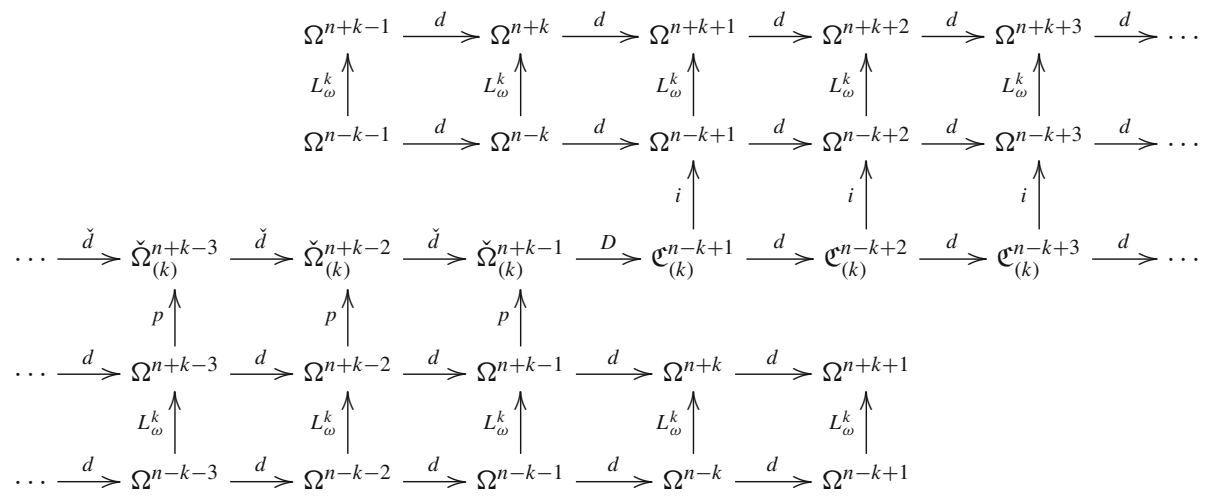

where $i$ denotes the inclusion and $p$ the natural projection, give rise to the following long exact sequence in cohomology:

$$
\begin{aligned}
& \cdots \stackrel{\check{f}_{n-k-2}}{\longrightarrow} H^{n-k-2}(M) \stackrel{L^{k}}{\longrightarrow} H^{n+k-2}(M) \stackrel{H(p)}{\longrightarrow} \check{H}_{(k)}^{n+k-2}(M) \\
& \stackrel{\check{f}_{n-k-1}}{\longrightarrow} H^{n-k-1}(M) \stackrel{L^{k}}{\longrightarrow} H^{n+k-1}(M) \stackrel{H(p)}{\longrightarrow} \check{H}_{(k)}^{n+k-1}(M) \\
& \stackrel{\check{f}_{n-k}}{\longrightarrow} H^{n-k}(M) \stackrel{L^{k}}{\longrightarrow} H^{n+k}(M) \stackrel{\check{f}_{n-k+1}}{\longrightarrow} \check{H}_{(k)}^{n+k}(M) \\
& \stackrel{H(i)}{\longrightarrow} H^{n-k+1}(M) \stackrel{L^{k}}{\longrightarrow} H^{n+k+1}(M) \stackrel{\check{f}_{n-k+2}}{\longrightarrow} \check{H}_{(k)}^{n+k+1}(M) \\
& \stackrel{H(i)}{\longrightarrow} H^{n-k+2}(M) \stackrel{L^{k}}{\longrightarrow} H^{n+k+2}(M) \stackrel{\check{f}_{n-k+3}}{\longrightarrow} \check{H}_{(k)}^{n+k+2}(M) \cdots
\end{aligned}
$$

Here $H(i)$ and $H(p)$ are the homomorphisms induced in cohomology by $i$ and $p$, respectively, and $\check{f}_{q}$ are the connecting homomorphisms, which are given as follows:

- for any $j \leq n+k-1$ and $[\alpha] \in \check{H}_{(k)}^{j}(M): \check{f}_{j-2 k+1}([\alpha])=[\beta]$, where $d \alpha=L_{\omega}^{k}(\beta)$;

- for any $j \geq n+k$ and $[\alpha] \in H^{j}(M): \check{f}_{j-2 k+1}([\alpha])=[d \beta]$, where $\alpha=L_{\omega}^{k}(\beta)$.

Let $\check{c}_{q}^{(k)}(M)$ be the dimension of $\check{H}_{(k)}^{q}(M)$ when it is finite. As in Sect. 2, using five-term exact sequences from (8), we arrive at the following result, that provides an extension of Proposition 2.5.

Proposition 3.1 Let $\left(M^{2 n}, \omega\right)$ be a symplectic manifold of finite type and let $1 \leq k \leq n$. Then, for every $0 \leq q \leq 2 n+2 k-1$, the following properties hold: 
(i) Finiteness and bounds for the numbers $\check{c}_{q}^{(k)}(M)$ : the group $\check{H}_{(k)}^{q}(M)$ is finite dimensional and its dimension $\check{c}_{q}^{(k)}(M)$ satisfies the inequalities

$$
b_{q-2 k+1}(M)-b_{q+1}(M) \leq \check{c}_{q}^{(k)}(M) \leq b_{q-2 k+1}(M)+b_{q}(M) .
$$

(ii) Symplectic manifolds satisfying the HLC: if $\left(M^{2 n}, \omega\right)$ satisfies the HLC then the lower bound in (9) is attained for every $q \geq n+k$, i.e.

$$
\check{c}_{q}^{(k)}(M)=b_{q-2 k+1}(M)-b_{q+1}(M), \quad q \geq n+k .
$$

(iii) Exact symplectic manifolds: if $\omega$ is exact then the upper bound in (9) is attained, i.e.

$$
\check{c}_{q}^{(k)}(M)=b_{q-2 k+1}(M)+b_{q}(M) .
$$

(iv) Poincaré lemma: if $U$ is the open unit disk in $\mathbb{R}^{2 n}$ with the standard symplectic form $\omega=\sum_{i=1}^{n} d x^{i} \wedge d x^{n+i}$, then $\check{c}_{0}^{(k)}(U)=1=\check{c}_{2 k-1}^{(k)}(U)$ and $\check{c}_{q}^{(k)}(U)=0$ for any other value of $q$.

Remark 3.2 By (ii) the lower bound in (9) is attained for every $q \geq n+k$ for symplectic manifolds satisfying the HLC. Similarly, it can be proved from (8) that if $\left(M^{2 n}, \omega\right)$ satisfies that all the maps $L^{k}: H^{n-k}(M) \longrightarrow H^{n+k}(M)$ are injective then $\check{c}_{q}^{(k)}(M)=b_{q}(M)-$ $b_{q-2 k}(M)$ for every $q \leq n+k-1$. In conclusion, if $L^{k}: H^{n-k}(M) \longrightarrow H^{n+k}(M)$ is an isomorphism for any $1 \leq k \leq n$ (for instance, if $\left(M^{2 n}, \omega\right)$ is a closed symplectic manifold satisfying the HLC) then the following equalities hold:

$$
\begin{aligned}
& \check{c}_{q}^{(k)}(M)=b_{q}(M)-b_{q-2 k}(M), \quad 0 \leq q \leq n+k-1 ; \\
& \check{c}_{q}^{(k)}(M)=b_{q-2 k+1}(M)-b_{q+1}(M), \quad n+k \leq q \leq 2 n+2 k-1 .
\end{aligned}
$$

Example 3.3 By Proposition 3.1 (iv) we have $\check{c}_{2 k-1}^{(k)}(U)=1$. Next we show the nonzero cohomology class generating $\check{H}_{(k)}^{2 k-1}(U)$. Let $\alpha=\sum_{i=1}^{n} x^{i} \wedge d x^{n+i}$. The $(2 k-1)$-form $\beta=\alpha \wedge \omega^{k-1}$ is $\breve{d}$-closed because $d \alpha=\omega$ and hence $d \beta=\omega^{k} \in L_{\omega}^{k}\left(\Omega^{0}(U)\right)$. Clearly, $\beta$ is not $\check{d}$-exact, because it is not $d$-exact. In conclusion, $[\beta]$ defines a nonzero cohomology class and $\check{H}_{(k)}^{2 k-1}(U)=\langle[\beta]\rangle$.

Remark 3.4 Notice that the generalized coeffective space $\hat{H}^{n-k+1}(M)$ is isomorphic to a quotient of $\check{H}_{(k)}^{n+k}(M)$; concretely,

$$
\hat{H}^{n-k+1}(M) \cong \frac{\check{H}_{(k)}^{n+k}(M)}{H^{n+k}(M) / L^{k}\left(H^{n-k}(M)\right)} .
$$

Let $\left(M^{2 n}, \omega\right)$ be a symplectic manifold of finite type. For every $1 \leq k \leq n$, we define

$$
\check{\chi}^{(k)}(M)=\sum_{i=0}^{2 n+2 k-1}(-1)^{i} \check{c}_{i}^{(k)}(M) .
$$

Let us write $\check{\chi}^{(k)}(M)=\check{\chi}_{+}^{(k)}(M)+\check{\chi}_{-}^{(k)}(M)$, where

$$
\check{\chi}_{+}^{(k)}(M)=\sum_{i=0}^{n+k-1}(-1)^{i} \check{c}_{i}^{(k)}(M), \quad \text { and } \quad \check{\chi}_{-}^{(k)}(M)=\sum_{i=n+k}^{2 n+2 k-1}(-1)^{i} \check{c}_{i}^{(k)}(M) .
$$


Proposition 3.5 Let $\left(M^{2 n}, \omega\right)$ be of finite type. For every $1 \leq k \leq n$ :

(i) $\check{\chi}^{(k)}(M)=0$; consequently, $\check{\chi}_{-}^{(k)}(M)=-\check{\chi}_{+}^{(k)}(M)$.

(ii) $\check{\chi}_{+}^{(k)}(M)=(-1)^{n+k+1}\left(\check{c}_{n+k}^{(k)}(M)-\hat{c}_{n-k+1}(M)\right)+\chi^{(k)}(M)$.

Proof Property (i) follows from (8) arguing similarly to the proof of Proposition 2.9.

For the proof of (ii), taking into account that $(-1)^{n+k+s} \breve{c}_{n+k+s}^{(k)}(M)=-(-1)^{n-k+s+1}$ $c_{n-k+s+1}^{(k)}(M)$ for $s \geq 1$, by Proposition 2.9 we get $\chi^{(k)}(M)+\check{\chi}_{-}^{(k)}(M)=(-1)^{n+k}\left(\check{c}_{n+k}^{(k)}(M)-\right.$ $\left.\hat{c}_{n-k+1}(M)\right)$. Since $\check{\chi}_{+}^{(k)}(M)=-\check{\chi}_{-}^{(k)}(M)$, relation (ii) follows.

Equality (ii) in the above proposition means that the behavior of the symplectic invariant $\check{\chi}_{+}^{(k)}(M)$ only depends on $\check{c}_{n+k}^{(k)}(M)-\hat{c}_{n-k+1}(M)$, because $\chi^{(k)}(M)$ is a topological invariant by Proposition 2.9. Moreover, one has the following characterization of the HLC in terms of $\check{\chi}_{+}^{(k)}(M)$, which in particular implies that the HLC is determined by the cohomology of the first half of the complexes (7).

Corollary 3.6 A symplectic manifold $\left(M^{2 n}, \omega\right)$ of finite type satisfies the HLC if and only if $\check{\chi}_{+}^{(k)}(M)=\chi^{(k)}(M)$ for every $1 \leq k \leq n$.

Proof By (10), a symplectic manifold satisfies the HLC if and only if $\hat{H}^{n-k+1}(M) \cong$ $\check{H}_{(k)}^{n+k}(M)$ for every $1 \leq k \leq n$. Therefore, if $M$ is of finite type then, $\left(M^{2 n}, \omega\right)$ satisfies the HLC if and only if $\hat{c}_{n-k+1}(M)=\check{c}_{n+k}^{(k)}(M)$ for every $1 \leq k \leq n$. By Proposition 3.5 (ii), this is equivalent to $\check{\chi}_{+}^{(k)}(M)=\chi^{(k)}(M)$ for every $1 \leq k \leq n$.

Remark 3.7 Tsai et al. [25, Theorem 3.1] have introduced elliptic differential complexes of filtered forms that extend the complex of primitive forms [27, Proposition 2.8] (see also [24] for recent progress). The difference with (7) is precisely that the second half of the complex is the image of the complex in [25] by the symplectic star operator (see Sect. 4), so in this sense the complex (7) can be thought as a coeffective version of the filtered complex.

On the other hand, [25, Theorem 4.2] gives long exact sequences that provide a resolution of the Lefschetz maps $L^{k}$. Comparing with (8), which also gives a resolution of the same Lefschetz maps, one immediately concludes that the cohomology $\check{H}_{(k)}^{*}(M)$ is isomorphic to the $(k-1)$-filtered cohomology as follows:

- $\check{H}_{(k)}^{n+k-s}(M) \cong F^{k-1} H_{+}^{n+k-s}(M)$, for $s=1, \ldots, n+k$,

- $\check{H}_{(k)}^{n+k+s}(M) \cong F^{k-1} H_{-}^{n+k-s-1}(M)$, for $s=0,1, \ldots, n+k-1$.

In particular, for any $k \geq 1$, one has the following isomorphism between the $(k-1)$-filtered cohomology group and the $k$-coeffective cohomology group

$$
F^{k-1} H_{-}^{n+k-s-1}(M) \cong H_{(k)}^{n-k+s+1}(M) \cong \check{H}_{(k)}^{n+k+s}(M), \quad 1 \leq s \leq n+k-1 .
$$

For $k=1$ we recover the isomorphisms proved in [10] between the extended coeffective cohomology of Eastwood and the primitive cohomology $P H=F^{0} H$ of Tseng and Yau [26, 27]. More generally, the isomorphism for any primitive cohomology group is as follows:

$$
\begin{aligned}
& P H_{\partial_{+}}^{q}(M) \cong F^{0} H_{+}^{q}(M) \cong \check{H}_{(1)}^{q}(M), \quad 0 \leq q \leq n-1 ; \\
& P H_{\partial_{-}}^{q}(M) \cong F^{0} H_{-}^{q}(M) \cong \check{H}_{(1)}^{2 n-q+1}(M) \cong H_{(1)}^{2 n-q}(M), \quad 0 \leq q \leq n-1 ; \\
& P H_{d d^{\Lambda}}^{n-k+1}(M) \cong F^{k-1} H_{+}^{n+k-1}(M) \cong \check{H}_{(k)}^{n+k-1}(M), \quad 1 \leq k \leq n ; \\
& P H_{d+d^{\Lambda}}^{n-k+1}(M) \cong F^{k-1} H_{-}^{n+k-1}(M) \cong \check{H}_{(k)}^{n+k}(M), \quad 1 \leq k \leq n .
\end{aligned}
$$


From now on, due to the above identifications, we will refer to the cohomology groups $\check{H}_{(k)}^{q}(M)$ as the filtered cohomology groups of $\left(M^{2 n}, \omega\right)$.

Notice that an analogous observation as Remark 2.10 is also valid for the filtered cohomologies. Hence, a similar result to Lemma 2.11 holds:

Lemma 3.8 Let $F:(M, \omega) \longrightarrow\left(M^{\prime}, \omega^{\prime}\right)$ be a diffeomorphism such that $F^{*}\left[\omega^{\prime}\right]=\lambda[\omega]$ for some nonzero $\lambda \in \mathbb{R}$. Then, for any $1 \leq k \leq n, \check{H}_{(k)}^{q}\left(M^{\prime}\right) \cong \check{H}_{(k)}^{q}(M)$ for every $0 \leq q \leq 2 n+2 k-1$.

A similar result to Proposition 2.12 for computation of the filtered cohomologies of certain solvmanifolds is also available:

Proposition 3.9 Let $(M=G / \Gamma, \omega)$ be a 2 n-dimensional symplectic solvmanifold satisfying the Mostow condition. Let $\mathfrak{g}$ be the Lie algebra of $G$ and let $\omega^{\prime} \in \bigwedge^{2} \mathfrak{g}^{*}$ be a left-invariant symplectic form representing the de Rham class $[\lambda \omega] \in H^{2}(M)$ for some $\lambda \neq 0$. Then, for any $1 \leq k \leq n$, the inclusion $\bigwedge^{*} \mathfrak{g}^{*} \hookrightarrow \Omega^{*}(M)$ induces isomorphisms $\check{H}_{(k)}^{q}(M, \omega) \cong \check{H}_{(k)}^{q}\left(\mathfrak{g}, \omega^{\prime}\right)$ for every $0 \leq q \leq 2 n+2 k-1$.

Remark 3.10 Tseng and Yau [26] introduced and studied more generally Bott-Chern and Aeppli type cohomologies using $d$ and $d^{\Lambda}$ for a symplectic manifold. A characterization of the HLC in the compact case from an à la Frölicher inequality is given in [3]. Note that for the Bott-Chern and Aeppli type symplectic cohomologies, a similar result to Proposition 3.9 is obtained in [19, Theorem 3] (see also [2, Theorem 2.31]) by using another argument.

\section{Relations with the symplectically harmonic cohomology}

In this section, we relate the symplectically harmonic cohomology with the cohomologies studied in the previous sections.

Let $\left(M^{2 n}, \omega\right)$ be a symplectic manifold of dimension $2 n$. The symplectic star operator *: $\Omega^{q}(M) \longrightarrow \Omega^{2 n-q}(M)$ is defined by

$$
\alpha \wedge(* \beta)=\Lambda^{q}(\Pi)(\alpha, \beta) \frac{\omega^{n}}{n !},
$$

for every $q$-forms $\alpha$ and $\beta$, where $\Pi$ is the bivector field dual to $\omega$, i.e. the natural Poisson structure associated with $\omega$.

Let $\delta: \Omega^{q}(M) \longrightarrow \Omega^{q-1}(M)$ be the operator given by $\delta \alpha=(-1)^{q+1} * d * \alpha$, for every $q$-form $\alpha$. Brylinski proved that $\delta=[i(\Pi), d]$, where $i(\cdot)$ denotes the interior product.

Definition 4.1 [7] A form $\alpha$ is called symplectically harmonic if $d \alpha=0=\delta \alpha$.

We denote by $\Omega_{\mathrm{hr}}^{q}(M)$ the linear space of symplectically harmonic $q$-forms. Unlike the Hodge theory, there are nonzero exact symplectically harmonic forms. Now, following Brylinski [7], one defines the symplectically harmonic cohomology

$$
H_{\mathrm{hr}}^{q}(M)=\frac{\Omega_{\mathrm{hr}}^{q}(M)}{\Omega_{\mathrm{hr}}^{q}(M) \cap \operatorname{im} d},
$$

for $0 \leq q \leq 2 n$. Hence, $H_{\mathrm{hr}}^{q}(M)$ is the subspace of the $q$ th de Rham cohomology group consisting of all the de Rham cohomology classes of degree $q$ containing a symplectically 
harmonic representative. By analogy with the Hodge theory, Brylinski [7] conjectured that any de Rham cohomology class admits a symplectically harmonic representative. Mathieu [20] (and independently Yan [29]) proved that Brylinski conjecture holds, namely $H_{\mathrm{hr}}^{q}(M, \omega)=$ $H^{q}(M)$ for every $0 \leq q \leq 2 n$, if and only if $\left(M^{2 n}, \omega\right)$ satisfies the HLC.

An important result is that for any symplectic manifold every de Rham cohomology class up to degree 2 admits a symplectically harmonic representative [29] (see also [16] for more general results), that is, $H_{\mathrm{hr}}^{q}(M)=H^{q}(M)$ for $q=0,1,2$. For every $q \leq n$, if we set

$$
P^{q}(M)=\left\{[\alpha] \in H^{q}(M) \mid L^{n-q+1}[\alpha]=0\right\},
$$

then $P^{q}(M) \subset H_{\mathrm{hr}}^{q}(M)$ [29]. Moreover, the following result (proved in [16, Corollary 2.4] and [28, Lemma 4.3]) gives a description of the spaces $H_{\mathrm{hr}}^{q}(M)$ :

Theorem 4.2 Let $\left(M^{2 n}, \omega\right)$ be a symplectic manifold of dimension $2 n$. Then,

$$
\begin{aligned}
& H_{\mathrm{hr}}^{q}(M)=P^{q}(M, \omega)+L\left(H_{\mathrm{hr}}^{q-2}(M)\right), \text { for } 0 \leq q \leq n \\
& H_{\mathrm{hr}}^{q}(M)=\operatorname{Im}\left\{L^{q-n}: H_{\mathrm{hr}}^{2 n-q}(M) \longrightarrow H^{q}(M)\right\}, \text { for } n+1 \leq q \leq 2 n .
\end{aligned}
$$

Next we suppose that $\left(M^{2 n}, \omega\right)$ is of finite type and denote by $h_{q}(M)$ the dimension of $H_{\mathrm{hr}}^{q}(M)$.

Example 4.3 Let $M^{n}$ be a manifold of dimension $n$ and of finite type, and let $\left(T^{*} M, \omega_{0}\right)$ be the cotangent bundle endowed with the standard symplectic form. Since $\omega_{0}$ is exact, the homomorphisms $L^{k}$ are identically zero, and by Theorem 4.2 we have

$h_{q}\left(T^{*} M, \omega_{0}\right)=b_{q}(M), \quad$ for $q \leq n, \quad$ and $h_{q}\left(T^{*} M, \omega_{0}\right)=0, \quad$ for $n+1 \leq q \leq 2 n$.

For the generalized coeffective cohomology, from Propositions 2.5 (iii) and 2.7 (iii) it follows that

$$
\hat{c}_{n-k+1}\left(T^{*} M, \omega_{0}\right)=b_{n-k+1}(M)
$$

and

$$
c_{q}^{(k)}\left(T^{*} M, \omega_{0}\right)=b_{q}(M)+b_{q+2 k-1}(M), \text { for } n-k+2 \leq q \leq 2 n .
$$

Furthermore, from Proposition 3.1 (iii) we get

$$
\check{c}_{q}^{(k)}\left(T^{*} M, \omega_{0}\right)=b_{q-2 k+1}(M)+b_{q}(M), \text { for } q \leq 2 n+2 k-1 .
$$

In the following result, we relate the generalized coeffective cohomology with the harmonic cohomology via the coeffective groups $\hat{H}^{1}(M), \ldots, \hat{H}^{n}(M)$.

Theorem 4.4 Let $\left(M^{2 n}, \omega\right)$ be a symplectic manifold of finite type. The following relation holds for every $k=1, \ldots, n$ :

$$
h_{n-k+1}(M)-h_{n+k+1}(M)=\hat{c}_{n-k+1}(M) .
$$

Proof By Theorem 4.2, $H_{\mathrm{hr}}^{n-k+1}(M)=P^{n-k+1}(M)+L\left(H_{\mathrm{hr}}^{n-k-1}(M)\right)$. Hence,

$$
\begin{aligned}
h_{n-k+1}(M)= & \operatorname{dim} P^{n-k+1}(M)+\operatorname{dim} L\left(H_{\mathrm{hr}}^{n-k-1}(M)\right) \\
& -\operatorname{dim}\left(P^{n-k+1}(M) \cap L\left(H_{\mathrm{hr}}^{n-k-1}(M)\right)\right) .
\end{aligned}
$$


It follows from (3) that $P^{n-k+1}(M)$ is isomorphic to the space $\hat{H}^{n-k+1}(M)$, and therefore, $\operatorname{dim} P^{n-k+1}(M)=\hat{c}_{n-k+1}(M)$. On the other hand,

$$
P^{n-k+1}(M) \cap L\left(H_{\mathrm{hr}}^{n-k-1}(M)\right)=\left.\operatorname{ker} L^{k}\right|_{L\left(H_{\mathrm{hr}}^{n-k-1}(M)\right)} \cdot
$$

Now,

$$
\begin{aligned}
h_{n-k+1}(M) & =\hat{c}_{n-k+1}(M)+\operatorname{dim} L\left(H_{\mathrm{hr}}^{n-k-1}(M)\right)-\operatorname{dim}\left(\left.\operatorname{ker} L^{k}\right|_{L\left(H_{\mathrm{hr}}^{n-k-1}(M)\right)}\right) \\
& =\hat{c}_{n-k+1}(M)+\operatorname{dim}\left(L^{k+1}\left(H_{\mathrm{hr}}^{n-k-1}(M)\right)\right) \\
& =\hat{c}_{n-k+1}(M)+h_{n+k+1}(M) .
\end{aligned}
$$

From Proposition 2.7, we get directly upper and lower bounds for the difference $h_{n-k+1}(M)-h_{n+k+1}(M)$. Moreover, the previous theorem, together with Proposition 2.9 and (11), provides further relations between the harmonic and the filtered cohomologies.

Next we derive some concrete relations of the harmonic cohomology with the groups $\check{H}_{(k)}^{q}(M)$.

Proposition 4.5 Let $\left(M^{2 n}, \omega\right)$ be a symplectic manifold of finite type. For every $1 \leq k \leq n$, we have

$$
0 \leq \check{c}_{n+k}^{(k)}(M)-\hat{c}_{n-k+1}(M) \leq b_{n+k}(M)-h_{n+k}(M),
$$

where the latter equality holds if and only if $L^{k}\left(H_{\mathrm{hr}}^{n-k}(M)\right)=L^{k}\left(H^{n-k}(M)\right)$.

Proof It follows from (10) that $\hat{c}_{n-k+1}(M)=\check{c}_{n+k}^{(k)}(M)-b_{n+k}(M)+\operatorname{dim} L^{k}\left(H^{n-k}(M)\right)$. Taking into account that $\operatorname{dim} L^{k}\left(H^{n-k}(M)\right) \geq h_{n+k}(M)$ by Theorem 4.2, we conclude the relation.

The following consequence will be useful later for symplectic manifolds of low dimension.

Corollary 4.6 Let $\left(M^{2 n}, \omega\right)$ be a symplectic manifold of finite type. Then:

$$
\begin{aligned}
\check{c}_{2 n}^{(n)}(M) & =b_{1}(M)+b_{2 n}(M)-h_{2 n}(M), \\
\check{c}_{2 n-1}^{(n-1)}(M) & =b_{2}(M)+b_{2 n-1}(M)-h_{2 n-1}(M)-h_{2 n}(M), \\
\check{c}_{2 n-2}^{(n-2)}(M) & =b_{2 n-2}(M)+h_{3}(M)-h_{2 n-2}(M)-h_{2 n-1}(M) .
\end{aligned}
$$

Proof Notice that $L^{k}\left(H_{\mathrm{hr}}^{n-k}(M)\right)=L^{k}\left(H^{n-k}(M)\right)$ is satisfied for $k=n, n-1$ and $n-2$ because $H_{\mathrm{hr}}^{q}(M)=H^{q}(M)$ for $q=0,1,2$. Hence, it suffices to apply Proposition 4.5 and use Theorem 4.4 to relate the coeffective numbers with the harmonic cohomology.

Next we show some other general properties that we will use later in the following sections.

Proposition 4.7 Let $\left(M^{2 n}, \omega\right)$ be a symplectic manifold of finite type. Then:

(i) $\hat{c}_{1}(M)=b_{1}(M)$, and $c_{q}^{(n)}(M)=b_{q}(M)$ for every $2 \leq q \leq 2 n$.

(ii) For any $1 \leq k \leq n, \check{c}_{q}^{(k)}(M)=c_{q-2 k+1}^{(k)}(M)$ for every $n+k+1 \leq q \leq 2 n+2 k-1$. 
Proof (i) is clear from the definition of the generalized coeffective cohomology for $k=n$ and from the long exact sequence (3). Equalities (ii) are direct from the definition of $\check{H}_{(k)}^{q}(M)$.

For closed manifolds one has additional relations. For instance, the numbers $\check{c}_{q}^{(k)}(M)$ satisfy certain duality (see [25, Proposition 4.8] for the corresponding duality for the filtered cohomology groups), whereas the harmonic number $h_{2 n-1}(M)$ is always even [16, Lemma 1.14]. The proof of these facts follows from the existence of the usual non-singular pairing $p([\alpha],[\beta])=\int_{M} \alpha \wedge \beta$, for $[\alpha] \in H^{q}(M)$ and $[\beta] \in H^{m-q}(M)$, valid on any closed $m$-dimensional manifold $M$. In the following proposition, we collect these results together with other properties.

Proposition 4.8 Let $\left(M^{2 n}, \omega\right)$ be a closed symplectic manifold. Then:

(i) For any $1 \leq k \leq n-1, c_{q}^{(k)}(M)=b_{q}(M)$ for every $2 n-2 k+1 \leq q \leq 2 n$.

(ii) For any $1 \leq k \leq n, \check{c}_{q}^{(k)}(M)=\check{c}_{2 n+2 k-q-1}^{(k)}(M)$ for every $0 \leq q \leq n+k-1$.

(iii) $h_{2 n}(M)=b_{2 n}(M)$, and $h_{2 n-1}(M)$ is always even.

(iv) $h_{n-1}(M)-\left(\check{c}_{n+1}^{(1)}(M)-\hat{c}_{n}(M)\right) \leq h_{n+1}(M) \leq h_{n-1}(M)$.

Proof By definition of the coeffective cohomology one always has that $c_{q}^{(k)}(M)=b_{q}(M)$ for any $1 \leq k \leq n-1$ and for every $q \geq 2 n-2 k+2$. Moreover, since $M$ is closed, $H^{2 n}(M)=$ $\left\langle\left[\omega^{n}\right]\right\rangle$ and $L^{k}: H^{2 n-2 k}(M) \longrightarrow H^{2 n}(M)$ is surjective, so the long exact sequence (3) implies $c_{2 n-2 k+1}^{(k)}(M)=b_{2 n-2 k+1}(M)$. This proves (i).

Property (ii) follows from [25, Proposition 4.8] taking into account the identifications given in Remark 3.7.

The proof of (iii) is a consequence of the fact that the rank of $L^{n-1}: H^{1}(M) \longrightarrow$ $H^{2 n-1}(M)$ is always an even number [16, Lemma 1.14].

To prove (iv), since $H_{\mathrm{hr}}^{n+1}(M)=L\left(H_{\mathrm{hr}}^{n-1}(M)\right)$, we have

$$
h_{n-1}(M)=h_{n+1}(M)+\operatorname{dim}\left(\operatorname{ker}\left\{L: H_{\mathrm{hr}}^{n-1}(M) \rightarrow H^{n+1}(M)\right\}\right) .
$$

Therefore,

$$
\begin{aligned}
h_{n-1}(M)-h_{n+1}(M) & =\operatorname{dim}\left(\operatorname{ker}\left\{L: H_{\mathrm{hr}}^{n-1}(M) \rightarrow H^{n+1}(M)\right\}\right) \\
& \leq \operatorname{dim}\left(\operatorname{ker}\left\{L: H^{n-1}(M) \rightarrow H^{n+1}(M)\right\}\right) \\
& =b_{n-1}(M)-\operatorname{dim}\left(\operatorname{im}\left\{L: H^{n-1}(M) \rightarrow H^{n+1}(M)\right\}\right) \\
& =b_{n+1}(M)-\operatorname{dim}\left(\operatorname{im}\left\{L: H^{n-1}(M) \rightarrow H^{n+1}(M)\right\}\right) \\
& =\check{c}_{n+1}^{(1)}(M)-\hat{c}_{n}(M),
\end{aligned}
$$

where the last equality follows from Remark 3.4.

Notice that Theorem 4.4 does not provide any relation for $h_{n+1}$, so (iv) in the above proposition provides upper and lower bounds for the harmonic number $h_{n+1}$ on closed symplectic manifolds.

Corollary 4.9 Let $\left(M^{2 n}, \omega\right)$ be a closed symplectic manifold. Then,

$$
\hat{c}_{1}(M)=\check{c}_{2 n}^{(n)}(M)=b_{1}(M) \text { and } \hat{c}_{2}(M)=b_{2}(M)-1 .
$$

Hence, the generalized coeffective cohomology groups (1) for $k=n$ and $n-1$, as well as the $n$-filtered cohomology groups are topological invariants. 
Proof The formulas for $\chi^{(n)}$ and $\chi^{(n-1)}$ given in Proposition 2.9 together with part (i) in Proposition 4.7 and Proposition 4.8 imply (12), so all the generalized $n$ - and $(n-1)$ coeffective numbers are topological. For the $n$-filtered cohomology it suffices to use Corollary 4.6 and the fact that $\check{c}_{q}^{(n)}=c_{q-2 n+1}^{(n)}$ for $2 n+1 \leq q \leq 4 n-1$.

We finish this section noticing that from Theorem 4.2 it follows directly that an analogous result to Lemmas 2.11 and 3.8 also holds for the symplectically harmonic cohomology [28, Proposition 1], and in the case of solvmanifolds satisfying the Mostow condition, a result similar to Propositions 2.12 and 3.9 is also valid (this was first observed in [28, Proposition 2], see also [16,17], for the class of symplectic nilmanifolds).

\section{Symplectic flexibility of closed manifolds}

In this section we focus on closed symplectic manifolds, for which we introduce a notion of flexibility for the generalized coeffective and filtered cohomologies, as analogous notions of the concept of harmonic flexibility introduced and studied in [16,29] and motivated by a question raised by Khesin and McDuff. Furthermore, we study their relations with the harmonic flexibility.

In what follows, $M$ will refer to a closed smooth manifold admitting symplectic forms.

Definition 5.1 A $2 n$-dimensional $M$ is said to be

(i) c-flexible, if $M$ possesses a continuous family of symplectic forms $\omega_{t}$, where $t \in[a, b]$, such that $\hat{c}_{n-k+1}\left(M, \omega_{a}\right) \neq \hat{c}_{n-k+1}\left(M, \omega_{b}\right)$ or $c_{q}^{(k)}\left(M, \omega_{a}\right) \neq c_{q}^{(k)}\left(M, \omega_{b}\right)$ for some $1 \leq k \leq n$ and $n-k+2 \leq q \leq 2 n$;

(ii) f-flexible, if $M$ possesses a continuous family of symplectic forms $\omega_{t}, t \in[a, b]$, such that $\check{c}_{q}^{(k)}\left(M, \omega_{a}\right) \neq \check{c}_{q}^{(k)}\left(M, \omega_{b}\right)$ for some $1 \leq k \leq n$ and $0 \leq q \leq 2 n+2 k-1$;

(iii) h-flexible, if $M$ possesses a continuous family of symplectic forms $\omega_{t}, t \in[a, b]$, such that $h_{q}\left(M, \omega_{a}\right) \neq h_{q}\left(M, \omega_{b}\right)$ for some $0 \leq q \leq 2 n$.

Notice that h-flexible manifolds are precisely the flexible manifolds in [16].

Since $H_{\mathrm{hr}}^{q}(M)=H^{q}(M)$ for $q=1,2$, we have $h_{2 n-q}(M)=\operatorname{dim}\left(\operatorname{Im}\left\{L^{n-q}: H^{q}(M) \longrightarrow\right.\right.$ $\left.\left.H^{2 n-q}(M)\right\}\right)$ for $q=1,2$ by Theorem 4.2. Now, if $\omega_{t}$ is a continuous family of symplectic structures on $M, t \in[a, b]$, then it is clear that

$$
h_{2 n-1}\left(M, \omega_{t}\right) \geq h_{2 n-1}\left(M, \omega_{a}\right) \text { and } h_{2 n-2}\left(M, \omega_{t}\right) \geq h_{2 n-2}\left(M, \omega_{a}\right),
$$

that is, these symplectically harmonic numbers satisfy a "lower-semicontinuous" property.

In the following result, we observe that an "upper-semicontinuous" property holds for all the coeffective and the filtered numbers.

Proposition 5.2 Let $\omega_{t}$ be a continuous family of symplectic structures on $M$, for $t \in[a, b]$. Then, for any $1 \leq k \leq n$ the following inequalities hold:

$$
\begin{aligned}
\hat{c}_{n-k+1}\left(M, \omega_{t}\right) & \leq \hat{c}_{n-k+1}\left(M, \omega_{a}\right), \\
c_{q}^{(k)}\left(M, \omega_{t}\right) & \leq c_{q}^{(k)}\left(M, \omega_{a}\right), \text { for every } n-k+2 \leq q \leq 2 n, \\
\check{c}_{n+k}^{(k)}\left(M, \omega_{t}\right) & \leq \check{c}_{n+k}^{(k)}\left(M, \omega_{a}\right), \text { for every } 0 \leq q \leq 2 n+2 k-1 .
\end{aligned}
$$

Proof It follows directly from the long exact sequences (3) and (8). 
Next we study relations among the three different types of flexibility. We begin in dimension four.

Theorem 5.3 Let $M$ be a 4-dimensional closed manifold. Then:

(i) $M$ is never c-flexible;

(ii) $M$ is f-flexible if and only if it is h-flexible.

Proof Since $n=2$, we need to study the coeffective numbers $c_{q}^{(1)}$ for $q=3,4, c_{q}^{(2)}$ for $q=2,3,4, \hat{c}_{1}$ and $\hat{c}_{2}$. Proposition 4.7 (i), Proposition 4.8 (i) and Corollary 4.9 imply that $c_{2}^{(2)}=b_{2}, c_{3}^{(1)}=c_{3}^{(2)}=b_{3}, c_{4}^{(1)}=c_{4}^{(2)}=b_{4}, \hat{c}_{1}=b_{1}$ and $\hat{c}_{2}=b_{2}-1$. Therefore, $M$ cannot be $c$-flexible and (i) is proved.

By Proposition 4.7 (ii) we have $\check{c}_{q}^{(k)}=c_{q-2 k+1}^{(k)}$ for $k=1,2$ and for any $3+k \leq q \leq 3+2 k$, so they are topological invariants. By the duality given in Proposition 4.8 (ii), it remains to study $\check{c}_{3}^{(1)}$ and $\check{c}_{4}^{(2)}$.

Since $h_{q}=b_{q}$ for $q=0,1,2$ and 4 , the first two equalities in Corollary 4.6 imply that $\check{c}_{4}^{(2)}=b_{1}$ and $\check{c}_{3}^{(1)}=b_{2}-b_{0}+b_{3}-h_{3}$, i.e.

$$
\check{c}_{3}^{(1)}=b_{1}+b_{2}-h_{3}-1 .
$$

Therefore, $M$ is $f$-flexible iff $M$ is $h$-flexible, since $\check{c}_{3}^{(1)}\left(M, \omega_{t}\right)$ varies along a family of symplectic forms $\omega_{t}$ iff $h_{3}\left(M, \omega_{t}\right)$ varies.

The previous proof shows that the fundamental relation between flexibilities on a 4dimensional manifold $M$ is

$$
\check{c}_{3}^{(1)}\left(M, \omega_{t}\right)=b_{1}(M)+b_{2}(M)-h_{3}\left(M, \omega_{t}\right)-1 .
$$

Corollary 5.4 Let $M$ be a 4-dimensional closed manifold. If the first Betti number $b_{1}(M) \leq 1$ then $M$ is not f-flexible.

Proof Proposition 4.8 (iii) implies that $h_{3}$ is an even number for any symplectic form. Since $h_{3} \leq b_{3}$ then $h_{3}=0$ and therefore $M$ cannot be flexible.

In particular, there do not exist simply connected closed 4-manifolds which are $f$-flexible.

On the other hand, Yan [29] proved that there are no $h$-flexible 4-dimensional nilmanifolds. Even more, one can see that the same holds in the bigger class of completely solvable solvmanifolds. For that, by the classification given in [5, Table 2] it remains to check that a solvmanifold based on the Lie algebra $d e^{1}=e^{13}, d e^{2}=-e^{23}, d e^{3}=d e^{4}=0$ is not $h$-flexible. In fact, any invariant symplectic structure is of the form $\omega=A e^{12}+B e^{13}+$ $C e^{23}+D e^{34}$, with $A D \neq 0$, and the number $h_{3}$ only depends on the element $[[\omega]]$ in $\mathbb{P}\left(H^{2}(M)\right.$ ) (see Remark 2.10), so we can suppose that $A=1$ and $B=C=0$ because $\left[e^{13}\right]=\left[e^{23}\right]=0$. Thus, it suffices to study the family $\omega_{t}=e^{12}+t e^{34}$, with $t \neq 0$. A direct calculation shows

$$
L_{\left[\omega_{t}\right]}\left(H^{1}(M)\right)=\left\langle\left[\omega_{t} \wedge e^{3}\right],\left[\omega_{t} \wedge e^{4}\right]\right\rangle=\left\langle\left[e^{123}\right],\left[e^{124}\right]\right\rangle=H^{3}(M),
$$

i.e. $h_{3}\left(\omega_{t}\right)=b_{3}$ for any $t \neq 0$, and therefore the solvmanifold is not $h$-flexible. Hence:

Proposition 5.5 Any 4-dimensional completely solvable solvmanifold is not c-flexible, fflexible or h-flexible. 
However, there exist 4-dimensional closed manifolds which are $h$-flexible, as it was proved in [29, Corollary 4.2] and [16, Proposition 3.2]. In fact, if $\left(M^{4}, \omega\right)$ is a closed symplectic manifold satisfying the conditions

(i) the homomorphism $L: H^{1}(M) \longrightarrow H^{3}(M)$ is trivial,

(ii) the cup product $H^{1}(M) \otimes H^{2}(M) \longrightarrow H^{3}(M)$ is non-trivial,

then $M$ is $h$-flexible. Since Gompf [13, Observation 7] proved the existence of 4-manifolds satisfying (i) and (ii), from Theorem 5.3 it follows that there exists 4-dimensional closed manifolds which are $f$-flexible. Moreover, taking symplectic products, we arrive at the following existence result:

Theorem 5.6 For each $n \geq 2$, there exist $2 n$-dimensional f-flexible closed manifolds. More precisely, there exists a $2 n$-dimensional closed manifold $M$ with a continuous family of symplectic forms $\omega_{t}$ such that the dimensions of the primitive cohomology groups $P H_{d+d^{\Lambda}}^{2}\left(M, \omega_{t}\right)$ and $P H_{d d^{\Lambda}}^{2}\left(M, \omega_{t}\right)$ vary with respect to $t$.

Proof Notice first that $\check{c}_{2 n-1}^{(n-1)}(M)=\operatorname{dim} P H_{d+d^{\Lambda}}^{2}(M)=\operatorname{dim} P H_{d d^{\Lambda}}^{2}(M)$, and by Corollary 4.6 we have $\check{c}_{2 n-1}^{(n-1)}(M)=b_{2}(M)+b_{1}(M)-h_{2 n-1}(M)-1$.

On the other hand, by [16, Proposition 5.3], we have the following formula for $h_{2 n-1}$ of a product $\left(M=N_{1} \times N_{2}, \omega=\omega_{1}+\omega_{2}\right)$ of two symplectic manifolds $\left(N_{1}, \omega_{1}\right)$ and $\left(N_{2}, \omega_{2}\right)$ of respective dimensions $n_{1}$ and $n_{2}$ :

$$
h_{2 n-1}(M)=h_{2 n_{1}-1}\left(N_{1}\right)+h_{2 n_{2}-1}\left(N_{2}\right) \text {, }
$$

where $n=n_{1}+n_{2}$.

Now, let $N_{1}$ be a 4-dimensional closed manifold such that $h_{3}$ varies along a continuous family of symplectic forms and let $N_{2}$ be, for instance, any compact Kähler manifold. Then, on the product manifold $M$, there is a continuous family of symplectic forms such that $h_{2 n-1}(M)$, and so $\check{c}_{2 n-1}^{(n-1)}(M)$, varies.

As we noticed above, there do not exist simply connected closed 4-manifolds which are $f$-flexible. By using a recent result by Cho [8], next we prove the existence of flexible simply connected closed manifolds in every dimension greater than or equal to six.

Theorem 5.7 For each $n \geq 3$, there exist $2 n$-dimensional simply connected closed manifolds which are f-flexible. More precisely, there exists a $2 n$-dimensional simply connected closed manifold $M$ with a continuous family of symplectic forms $\omega_{t}$ such that the dimensions of the primitive cohomology groups $P H_{d+d^{\Lambda}}^{3}\left(M, \omega_{t}\right)$ and $P H_{d d^{\Lambda}}^{3}\left(M, \omega_{t}\right)$ vary with respect to $t$. Moreover, the manifold $M$ is homotopy equivalent to some Kähler manifold.

Proof Let us first recall that Cho proves in [8, Theorem 1.3] the existence of a compact Kähler manifold $(X, \omega)$ with $\operatorname{dim}_{\mathbb{C}} X=3$ such that

(1) $X$ is simply connected,

(2) the odd Betti numbers vanish, i.e. $b_{2 k+1}(X)=0$ for every integer $k \geq 0$,

(3) $X$ admits a symplectic form $\sigma$ such that $(X, \sigma)$ does not satisfy the HLC, and

(4) $\sigma$ is deformation equivalent to the Kähler form $\omega$, that is, there is a path $\left\{\omega_{t}\right\}_{0 \leq t \leq 1}$ of symplectic forms such that $\omega_{0}=\omega$ and $\omega_{1}=\sigma$.

These properties imply that $h_{4}\left(X, \omega_{t}\right)$ varies with $t$, and the manifold $X$ is $h$-flexible. One can see that $\hat{c}_{3}=0$ and $c_{4}^{(1)}=b_{2}-1$ for any symplectic form on $X$, so it is not $c$-flexible, 
but $X$ is $f$-flexible because $\check{c}_{4}^{(1)}=b_{2}-h_{4}$. Hence $X$ provides an example in dimension 6 . Next we consider this manifold to prove that there are simply connected closed manifolds in every higher dimension which are $f$-flexible.

Let $M$ be a closed symplectic manifold of dimension $2 n$. From Remark 3.7 and Proposition 4.8 (ii) for $k=n-2$ and $q=2 n-3$, we notice that $\operatorname{dim} P H_{d+d^{\Lambda}}^{3}(M)=\check{c}_{2 n-2}^{(n-2)}(M)=$ $\check{c}_{2 n-3}^{(n-2)}(M)=\operatorname{dim} P H_{d d^{\Lambda}}^{3}(M)$. On the other hand, by Corollary 4.6

$$
\check{c}_{2 n-2}^{(n-2)}(M)=b_{2 n-2}(M)+h_{3}(M)-h_{2 n-2}(M)-h_{2 n-1}(M) .
$$

Suppose that $M$ is a product of two symplectic manifolds $\left(N_{1}, \omega_{1}\right)$ and $\left(N_{2}, \omega_{2}\right)$ of respective dimensions $n_{1}$ and $n_{2}$. By [16, Proposition 5.3], we have the following formulas for the harmonic numbers $h_{2 n-1}$ and $h_{2 n-2}$ of the manifold $\left(M=N_{1} \times N_{2}, \omega=\omega_{1}+\omega_{2}\right)$ :

$$
\begin{aligned}
& h_{2 n-1}(M)=h_{2 n_{1}-1}\left(N_{1}\right)+h_{2 n_{2}-1}\left(N_{2}\right), \\
& h_{2 n-2}(M)=h_{2 n_{1}-2}\left(N_{1}\right)+h_{2 n_{1}-1}\left(N_{1}\right) h_{2 n_{2}-1}\left(N_{2}\right)+h_{2 n_{2}-2}\left(N_{2}\right) .
\end{aligned}
$$

where $n=n_{1}+n_{2}$.

Now, let $N_{1}=X$ be the 6-dimensional simply connected closed manifold described above, and let $N_{2}=\mathbb{C P}^{n}$ endowed with the standard Kähler structure defined by its natural complex structure and the Fubini-Study metric. Hence, the manifold $M$ is a simply connected closed manifold of dimension $2 n=2\left(3+n_{2}\right) \geq 8$. Since all the odd Betti numbers of $N_{1}$ and $N_{2}$ vanish, the manifold $M$ also has all its odd Betti numbers equal to zero. This implies that $h_{3}(M)=0, h_{2 n-1}(M)=0$ and $h_{2 n-2}(M)=h_{4}(X)+1$ by $(15)$, because $h_{2 n_{2}-2}\left(N_{2}\right)=$ $b_{2 n_{2}-2}\left(\mathbb{C P}^{n_{2}}\right)=1$. Moreover, by Künneth formula one arrives at $b_{2 n-2}(M)=b_{4}(X)+1$. Therefore, equality (14) reduces to

$$
\check{c}_{2 n-2}^{(n-2)}(M)=b_{4}(X)-h_{4}(X) .
$$

By the properties of $X$ described above, we conclude that on the product manifold $M$, there is a continuous family of symplectic forms such that $\check{c}_{2 n-2}^{(n-2)}(M)$ varies.

From the proofs of Theorems 5.6 and 5.7, the resulting symplectic manifolds are also $h$-flexible. It is unclear if $f$-flexibility is implied by $h$-flexibility in dimension higher than or equal to 8 (see Proposition 5.11 for the general relation). In contrast, in six dimensions we have:

\section{Theorem 5.8 Let $M$ be a 6-dimensional closed manifold. Then:}

(i) If $M$ is c-flexible then $M$ is f-flexible and h-flexible.

(ii) If $M$ is not $C$-flexible then, $M$ is f-flexible if and only if it is h-flexible.

Proof Since $n=3$, the coeffective numbers to be studied are: $c_{q}^{(1)}$ for $q=4,5,6, c_{q}^{(2)}$ for $q=3,4,5,6, c_{q}^{(3)}$ for $q=2,3,4,5,6$, and $\hat{c}_{1}, \hat{c}_{2}, \hat{c}_{3}$. Corollary 4.9 implies that $\hat{c}_{1}, \hat{c}_{2}$ and all $c_{q}^{(k)}$ for $k=2,3$ are topological invariants. Moreover, by Proposition 4.8 (i) we have $c_{q}^{(1)}=b_{q}$ for $q=5,6$. Now, the formula for $\chi^{(1)}$ given in Proposition 2.9 implies that

$$
\hat{c}_{3}=c_{4}^{(1)}+b_{3}-b_{2}-b_{1}+1 .
$$

Therefore, $M$ is c-flexible if and only if $M$ possesses a continuous family of symplectic forms $\omega_{t}, t \in[a, b]$, such that $\hat{c}_{3}\left(M, \omega_{a}\right) \neq \hat{c}_{3}\left(M, \omega_{b}\right)$. 
By Proposition 4.7 (ii) we have $\check{c}_{q}^{(k)}=c_{q-2 k+1}^{(k)}$ for $k=1,2,3$ and for any $4+k \leq q \leq$ $5+2 k$, so they are topological invariants except possibly $\check{c}_{5}^{(1)}$, which satisfies

$$
\check{c}_{5}^{(1)}=c_{4}^{(1)} \text {. }
$$

By the duality given in Proposition 4.8 (ii), it remains to study $\check{c}_{4}^{(1)}$ and $\check{c}_{5}^{(2)}$.

Since $h_{q}=b_{q}$ for $q=0,1,2,6$, the equalities in Corollary 4.6 together with Theorem 4.4 imply the following relations

$$
\hat{c}_{3}=h_{3}-h_{5}, \quad \hat{c}_{3}=\check{c}_{4}^{(1)}+h_{4}-b_{2}, \quad \check{c}_{5}^{(2)}=-h_{5}+b_{2}+b_{1}-1 .
$$

Therefore, the fundamental equalities that relate the different cohomologies for closed 6-dimensional manifolds are (16)-(18). Now, using these relations, a direct argument shows (i) and (ii).

Corollary 5.9 A 6-dimensional closed manifold is f-flexible if and only if it is h-flexible.

Remark 5.10 Notice that there exist closed 6-dimensional manifolds which are $f$-flexible and $h$-flexible, but not $c$-flexible; that is to say, the converse to (i) in Theorem 5.8 does not hold in general. Explicit examples of nilmanifolds satisfying this are given in Sect. 6.

In higher dimension, we have the following result:

Proposition 5.11 Let $M$ be a closed manifold of dimension $2 n \geq 8$. If $M$ is c-flexible then $M$ is f-flexible or h-flexible.

Proof If $M$ possesses a continuous family of symplectic forms $\omega_{t}, t \in[a, b]$, such that $c_{q}^{(k)}\left(M, \omega_{a}\right) \neq c_{q}^{(k)}\left(M, \omega_{b}\right)$ for some $1 \leq k \leq n$ and $n-k+2 \leq q \leq 2 n$, then it is clear that $M$ is $f$-flexible by Proposition 4.7(ii).

If $M$ possesses a continuous family of symplectic forms $\omega_{t}, t \in[a, b]$, such that $\hat{c}_{n-k+1}\left(M, \omega_{a}\right) \neq \hat{c}_{n-k+1}\left(M, \omega_{b}\right)$ for some $1 \leq k \leq n$, then Theorem 4.4 implies that $h_{n-k+1}\left(M, \omega_{a}\right) \neq h_{n-k+1}\left(M, \omega_{b}\right)$ or $h_{n+k+1}\left(M, \omega_{a}\right) \neq h_{n+k+1}\left(M, \omega_{b}\right)$, therefore $M$ is h-flexible.

\section{Symplectic 6-dimensional nilmanifolds}

In this section, we present a complete study of the dimensions of the harmonic, coeffective and filtered cohomology groups of 6-dimensional symplectic nilmanifolds, see Table 1 below.

The symplectically harmonic numbers $h_{4}$ and $h_{5}$ were first computed in [16], whereas $h_{3}$ was obtained in [17] (see Remark 6.2 for corrections). As a consequence of our study, we describe all $c$-flexible, $h$-flexible or $f$-flexible 6-dimensional nilmanifolds.

In the proof of Theorem 5.8 we found that for 6-dimensional closed symplectic manifolds the fundamental equalities that relate the different cohomologies are (16)-(18). In addition, since the Euler characteristic of a nilmanifold vanishes, we have that $b_{3}=2\left(b_{2}-b_{1}+1\right)$. Therefore, relations (16)-(18) for 6-dimensional symplectic nilmanifolds are

$$
\begin{aligned}
\hat{c}_{3} & =c_{4}^{(1)}+b_{2}-3 b_{1}+3, \quad h_{3}=\hat{c}_{3}+h_{5}, \quad \check{c}_{4}^{(1)}=\hat{c}_{3}-h_{4}+b_{2}, \quad \check{c}_{5}^{(1)}=c_{4}^{(1)}, \\
\check{c}_{5}^{(2)} & =-h_{5}+b_{2}+b_{1}-1 .
\end{aligned}
$$


Recall that $c_{4}^{(1)}, \check{c}_{4}^{(1)}$ and $\check{c}_{5}^{(2)}$ are by Remark 3.7 dimensions of primitive cohomology groups; concretely, $c_{4}^{(1)}=\operatorname{dim} P H_{\partial_{-}}^{2}\left(=\operatorname{dim} P H_{\partial_{+}}^{2}\right), \check{c}_{4}^{(1)}=\operatorname{dim} P H_{d+d^{\Lambda}}^{3}\left(=\operatorname{dim} P H_{d d^{\Lambda}}^{3}\right)$ and $\check{c}_{5}^{(2)}=\operatorname{dim} P H_{d+d^{\Lambda}}^{2}\left(=\operatorname{dim} P H_{d d^{\Lambda}}^{2}\right)$.

It follows from Propositions 2.12 and 3.9 that the calculation of all the cohomology groups reduces to the Lie algebra level. In Table 1 nilmanifolds of dimension 6 admitting symplectic structure appear lexicographically with respect to the triple $\left(b_{1}, b_{2}, 6-s\right)$, where $b_{1}$ and $b_{2}$ are the Betti numbers (first two columns in the table) and $s$ is the step length (third column). The fourth column contains the description of the structure of the nilmanifold; for instance, the notation $(0,0,12,13,14,15)$ means that there exists a basis $\left\{e^{i}\right\}_{i=1}^{6}$ of (invariant) 1 -forms such that

$$
d e^{1}=d e^{2}=0, \quad d e^{3}=e^{1} \wedge e^{2}, \quad d e^{4}=e^{1} \wedge e^{3}, \quad d e^{5}=e^{1} \wedge e^{4}, \quad d e^{6}=e^{1} \wedge e^{5} .
$$

The next columns show the dimensions of the non-trivial harmonic, coeffective and filtered cohomology groups, that is, $h_{k}(k=3,4,5), \hat{c}_{3}, c_{4}^{(1)}\left(=\check{c}_{5}^{(1)}\right), \check{c}_{4}^{(1)}$ and $\check{c}_{5}^{(2)}$. Moreover, the columns contain all the possible values when $\omega$ runs over the space $\mathcal{S}$ of all invariant symplectic structures on the nilmanifold.

The last column shows the dimension of the space $\mathcal{S}$, however the cohomology groups only depend on the cohomology class of the symplectic form. This fact allows to reduce calculations to a smaller number of parameters, and furthermore by Remark 2.10, we can always normalize one of the nonzero coefficients that parametrize the classes of the symplectic forms.

When there are variations in the dimensions of the cohomology groups, they appear in the table written accordingly to the lower-semicontinuous property (13) of the harmonic numbers $h_{4}$ and $h_{5}$, or to the upper-semicontinuous property of $\hat{c}_{3}, c_{4}^{(1)}, \check{c}_{4}^{(1)}$ and $\check{c}_{5}^{(2)}$ (see Proposition 5.2). Notice that the harmonic number $h_{3}$ does not satisfy any lower- or uppersemicontinuous property. Moreover, the variations in the dimensions of the cohomology groups are in correspondence (in the sense that we explain in Example 6.3 below), except for the nilmanifolds $(0,0,0,12,13,23)$ and $(0,0,0,12,13,14+23)$ (see Example 6.4 for details on the latter).

The following result is a direct consequence of Table 1 .

Theorem 6.1 (i) There exist seven nilmanifolds of dimension 6 that are c-flexible (and therefore f-flexible and h-flexible).

(ii) There exist three nilmanifolds of dimension 6 that are f-flexible and h-flexible, but not c-flexible.

In conclusion, there exist ten 6-dimensional nilmanifolds that are f-flexible and h-flexible.

Notice that from (ii) it follows that the converse of Theorem 5.8 does not hold, that is, $c$-flexibility is the strongest condition.

Remark 6.2 In $[16,17]$ the following symplectically harmonic numbers need correction:

- for $(0,0,12,13,14,15)$ the number $h_{4}$ is equal to 2 (not 3 );

- for $(0,0,12,13,14,23+15)$ the number $h_{3}$ is equal to 3 (not 2 );

- for $(0,0,0,12,14,15+23)$ the number $h_{3}$ is equal to 5 (not 4$)$;

- for $(0,0,0,0,12,14+25)$ the number $h_{4}$ is equal to 4 (not 3 ).

Example 6.3 Let us consider the 6-dimensional nilmanifold $(0,0,0,12,14,15+23+24)$. According to Table 1 , this manifold is $c$-flexible, $f$-flexible and $h$-flexible. In fact, consider 


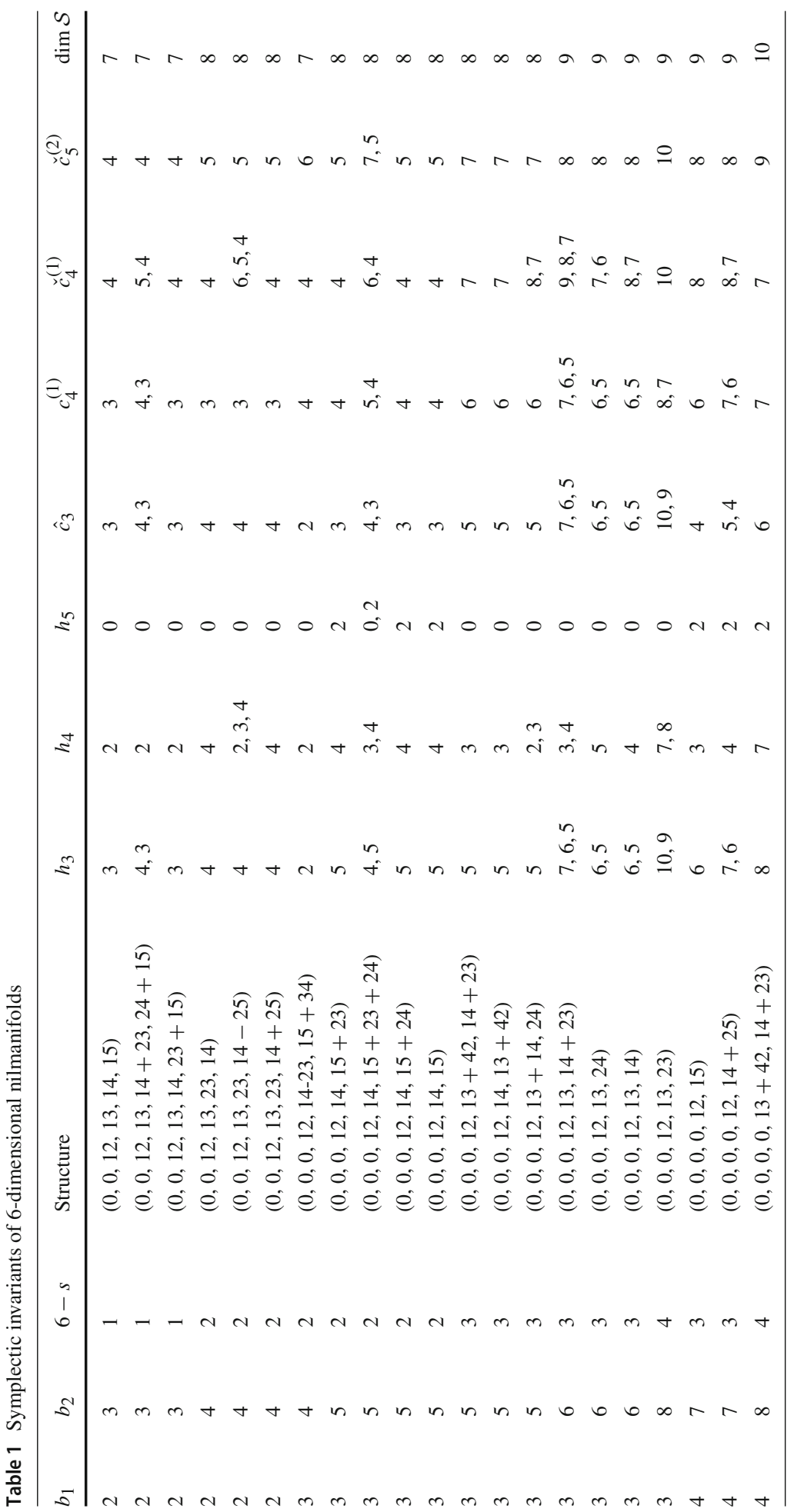




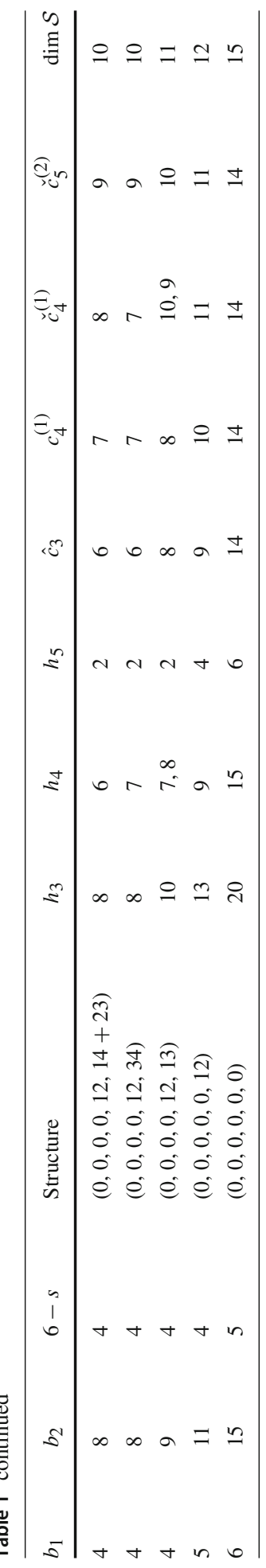

\section{를 Springer}


the following continuous family of symplectic structures

$$
\left[\omega_{t}\right]=(1-\cos t)\left[e^{13}\right]-\cos t\left[e^{16}+e^{25}-e^{34}\right]+(1-\cos t)\left[e^{26}-e^{45}\right], \quad t \in \mathbb{R} .
$$

This family was constructed first in $[16,17]$ to show $h$-flexibility, and the symplectic structures $\omega_{t=0}$ and $\omega_{t=\frac{\pi}{2}}$ were considered in [27] concerning the dimension of the primitive group $P H_{\partial_{-}}^{2}$, i.e. $c_{4}^{(1)}$.

This 6-dimensional nilmanifold is the only one where all the non-trivial coeffective, harmonic and primitive numbers vary. In Table 1 the variations are in correspondence as follows:

- $h_{3}\left(\omega_{2 \pi k}\right)=4, h_{4}\left(\omega_{2 \pi k}\right)=3, h_{5}\left(\omega_{2 \pi k}\right)=0, \hat{c}_{3}\left(\omega_{2 \pi k}\right)=4, c_{4}^{(1)}\left(\omega_{2 \pi k}\right)=\check{c}_{5}^{(1)}\left(\omega_{2 \pi k}\right)=$ $5, \check{c}_{4}^{(1)}\left(\omega_{2 \pi k}\right)=6, \check{c}_{5}^{(2)}\left(\omega_{2 \pi k}\right)=7$, for any integer $k$;

- $h_{3}\left(\omega_{t}\right)=5, h_{4}\left(\omega_{t}\right)=4, h_{5}\left(\omega_{t}\right)=2, \hat{c}_{3}\left(\omega_{t}\right)=3, c_{4}^{(1)}\left(\omega_{t}\right)=\check{c}_{5}^{(1)}\left(\omega_{t}\right)=4, \check{c}_{4}^{(1)}\left(\omega_{t}\right)=4$, $\check{c}_{5}^{(2)}\left(\omega_{t}\right)=5$, for $t \neq 2 \pi k$.

Example 6.4 Let us consider the 6-dimensional nilmanifold ( $0,0,0,12,13,14+23)$. The de Rham class of any symplectic form is given by

$$
[\omega]=A\left[e^{14}\right]+B\left[e^{15}\right]+C\left[e^{24}\right]+D\left[e^{35}\right]+E\left[e^{16}+e^{25}\right]+F\left[e^{16}-e^{34}\right],
$$

where $(E+F)(C D+E F) \neq 0$. Direct computations show that $\hat{c}_{3}$ and $h_{4}$ vary as follows:

$$
\hat{c}_{3}=\left\{\begin{array}{ll}
7, & \text { if } D=E+2 F=0, \\
6, & \text { if } D=0, E+2 F \neq 0, \\
5, & \text { if } D \neq 0,
\end{array} \quad h_{4}= \begin{cases}3, & \text { if }(E+F)^{2}=C D+E F, \\
4, & \text { if } \quad(E+F)^{2} \neq C D+E F .\end{cases}\right.
$$

This nilmanifold satisfies $b_{2}=3 b_{1}-3$, so (19) implies that $\check{c}_{5}^{(1)}=c_{4}^{(1)}=\hat{c}_{3}$. Moreover, $h_{5}=0$ from which we get that $\check{c}_{5}^{(2)}=8$ and $h_{3}=\hat{c}_{3}$. Hence, using that $\check{c}_{4}^{(1)}=b_{2}-h_{4}+\hat{c}_{3}$ by (19), we arrive at

$$
\check{c}_{4}^{(1)}= \begin{cases}9, & \text { if } D=E+2 F=0, \\ 8, & \text { if } D=0, E+2 F \neq 0, \text { or } D \neq 0,(E+F)^{2}=C D+E F, \\ 7, & \text { if } D \neq 0,(E+F)^{2} \neq C D+E F .\end{cases}
$$

As a consequence, concrete families can be constructed. Let us consider the two-parametric family

$$
\left[\omega_{t, s}\right]=t\left[e^{35}\right]+(s+2)\left[e^{16}+e^{25}\right]-\left[e^{16}-e^{34}\right],
$$

where $t, s \geq 0$. Then, the variations of the dimensions are:

- $h_{3}\left(\omega_{0,0}\right)=\hat{c}_{3}\left(\omega_{0,0}\right)=c_{4}^{(1)}\left(\omega_{0,0}\right)=\check{c}_{5}^{(1)}\left(\omega_{0,0}\right)=7$ and $\check{c}_{4}^{(1)}\left(\omega_{0,0}\right)=9$;

- $h_{3}\left(\omega_{t, 0}\right)=\hat{c}_{3}\left(\omega_{t, 0}\right)=c_{4}^{(1)}\left(\omega_{t, 0}\right)=\check{c}_{5}^{(1)}\left(\omega_{t, 0}\right)=5$ and $\check{c}_{4}^{(1)}\left(\omega_{t, 0}\right)=7$, for $t>0$;

- $h_{3}\left(\omega_{0, s}\right)=\hat{c}_{3}\left(\omega_{0, s}\right)=c_{4}^{(1)}\left(\omega_{0, s}\right)=\check{c}_{5}^{(1)}\left(\omega_{0, s}\right)=6$ and $\check{c}_{4}^{(1)}\left(\omega_{0, s}\right)=8$, for $s>0$.

On the other hand, if we consider the family

$$
\left[\omega_{t}\right]=\left[e^{24}\right]+\left[e^{35}\right]+t\left[e^{16}+e^{25}\right]+\left[e^{16}-e^{34}\right],
$$

where $t \geq 0$, then the variations are:

- $h_{4}\left(\omega_{0}\right)=3$ and $\check{c}_{4}^{(1)}\left(\omega_{0}\right)=8$; 
- $h_{4}\left(\omega_{t}\right)=4$ and $\check{c}_{4}^{(1)}\left(\omega_{t}\right)=7$, for $t>0$.

For compact Kähler manifolds $\left(M^{2 n}, \omega\right)$ and for every $q \geq n+1$, the coeffective cohomology group $H_{(1)}^{q}(M)$ is isomorphic to the $[\omega]$-truncated qth de Rham group $\widetilde{H}_{[\omega]}^{q}(M)=\left\{[\alpha] \in H^{q}(M) \mid[\alpha] \cup[\omega]=0\right\}$, although Fernández, Ibáñez and de León showed that this is no longer true for arbitrary compact symplectic manifolds [11]. Kasuya [18] has studied certain symplectic aspherical (non-Kähler) manifolds for which

$$
H_{(1)}^{q}(M) \cong \widetilde{H}_{[\omega]}^{q}(M) \text { for every } q \geq n+1 .
$$

For 6-dimensional symplectic nilmanifolds, one has the following result, which suggests that such isomorphism might be closely related to a low step of nilpotency:

Proposition 6.5 Let $M$ be a symplectic s-step nilmanifold of dimension 6.

(i) If $s \leq 2$, then there exists a symplectic form on $M$ satisfying (20).

(ii) If $s=5$ then (20) is never satisfied.

Proof Since $n=3$ we only need to consider $q=4$. Thus, (20) holds if and only if $c_{4}^{(1)}(M)=$ $\operatorname{dim} \widetilde{H}_{[\omega]}^{4}(M)=b_{2}(M)-1$, the latter equality coming from the fact that $L\left(H^{4}(M)\right)=$ $H^{6}(M)$. Now, the result is a direct consequence of Table 1 .

Notice that there are several 3-step and several 4-step symplectic nilmanifolds of dimension 6 satisfying (20). In fact, all the cases in Table 1 where $c_{4}^{(1)}=b_{2}-1$ have such property.

Remark 6.6 Concerning other (non-primitive) cohomology groups, we recall that in [2, Table 3] the dimensions of the symplectic Bott-Chern and Aeppli cohomologies for a particular choice of symplectic structure on each 6-dimensional nilmanifold have been computed.

\section{A symplectic 8-dimensional solvmanifold}

In this section we show a closed manifold of dimension 8 that is $c$-flexible, $f$-flexible and h-flexible.

Let us consider the 8-dimensional compact solvmanifold $M=S / \Gamma$, where $S$ is a simply connected completely solvable Lie group of dimension 8 defined by left-invariant 1 -forms $\left\{e^{i}, 1 \leq i \leq 8\right\}$ such that

$$
\begin{aligned}
d e^{1}=d e^{2} & =d e^{3}=0, \quad d e^{4}=-e^{12}, \quad d e^{5}=-e^{13}, \quad d e^{6}=-e^{14}, \\
d e^{7} & =-e^{17}, \quad d e^{8}=e^{18} .
\end{aligned}
$$

Using [15], the Betti numbers of $M$ are $b_{1}(M)=3, b_{2}(M)=7, b_{3}(M)=11$ and $b_{4}(M)=$ 12 (see [11] for a description of the de Rham cohomology groups of $M$ ).

The de Rham cohomology class of a generic symplectic 2-form $\omega$ on $M$ is given by

$$
[\omega]=A\left[e^{15}\right]+B\left[e^{16}\right]+C\left[e^{23}\right]+D\left[e^{24}\right]+E\left[e^{25}+e^{34}\right]+F\left[e^{35}\right]+G\left[e^{78}\right],
$$

where the coefficients $A, \ldots, G \in \mathbb{R}$ satisfy $B G\left(E^{2}-D F\right) \neq 0$. Notice that by Remark 2.10 , we can suppose without loss of generality that for instance $G=1$. Also notice that Propositions 2.12 and 3.9 allow us to reduce the computation of all the symplectic cohomology groups to the level of the Lie algebra $\mathfrak{s}$ of $S$. 
The generalized coeffective cohomology groups for $k=3,4$ provide no flexibility since they are topological invariants. A direct calculation shows that the $c$-flexibility depends only on $\hat{c}_{3}$ and, moreover, $\hat{c}_{3}=8$ or 9 depending on $F \neq 0$ or $F=0$, respectively.

Next we show that $\hat{c}_{3}$ is also a key ingredient to obtain the other types of flexibility. Using Theorem 4.4, we have that $h_{3}-h_{7}=\hat{c}_{3}$. It turns out that the map $L^{3}: H^{1} \longrightarrow H^{7}$ is identically zero, so $h_{7}=0$ and therefore $h_{3}=\hat{c}_{3}$. On the other hand, according to Corollary 4.6, $\check{c}_{6}^{(2)}=b_{6}+h_{3}-h_{6}-h_{7}$, thus $\check{c}_{6}^{(2)}=h_{3}+b_{2}-h_{6}$, or equivalently, $\check{c}_{6}^{(2)}=\hat{c}_{3}+\operatorname{dim} \operatorname{ker}\left\{L^{2}: H^{2} \longrightarrow H^{6}\right\}$. A direct calculation shows that the dimension of the kernel of the latter $L^{2}$-map is independent of the symplectic form and it is equal to 2 , therefore $\check{c}_{6}^{(2)}=\hat{c}_{3}+2$. Recall that $\check{c}_{6}^{(2)}$ is by Remark 3.7 the dimension of the primitive cohomology group $P H_{d+d^{\Lambda}}^{3} \cong P H_{d d^{\Lambda}}^{3}$.

From this general study one concludes that the closed manifold $M$ is $C$-flexible, $f$-flexible and $h$-flexible. For instance, if we consider the following family of symplectic forms

$$
\omega_{t}=e^{15}+e^{16}+t e^{23}-t e^{24}+e^{25}+e^{34}+t e^{35}+e^{78}, \quad t \in \mathbb{R},
$$

then:

- $\hat{c}_{3}\left(\omega_{0}\right)=h_{3}\left(\omega_{0}\right)=9$, and $\check{c}_{6}^{(2)}\left(\omega_{0}\right)=11$;

- $\hat{c}_{3}\left(\omega_{t}\right)=h_{3}\left(\omega_{t}\right)=8$, and $\check{c}_{6}^{(2)}\left(\omega_{t}\right)=10$, for any $t \neq 0$.

Remark 7.1 The symplectic manifold $\left(M, \omega_{0}\right)$ is considered in [11] (see also [12, page 288]) as an example of a compact symplectic manifold of dimension 8 for which (20) does not hold. However, the 1-coeffective cohomology groups were wrongly obtained and the conclusion is not correct. In fact, one can prove that (20) holds for any $t \in \mathbb{R}$, in particular for $t=0$.

On the other hand, by Remark 2.14, for any symplectic manifold $\left(M^{2 n}, \omega\right)$ satisfying the HLC (in particular, for any compact Kähler manifold) the $k$-coeffective cohomology group $H_{(k)}^{q}(M)$ is isomorphic to the $\left[\omega^{k}\right]$-truncated de Rham group $\widetilde{H}_{\left[\omega^{k}\right]}^{q}(M)=\left\{[\alpha] \in H^{q}(M) \mid\right.$ $\left.[\alpha] \cup\left[\omega^{k}\right]=0\right\}$, for any $q \geq n-k+2$ and $1 \leq k \leq n$. It is a natural question if such isomorphisms hold for any $k$ for arbitrary symplectic manifolds. A detailed study of the symplectic manifolds $\left(M, \omega_{t}\right)$ above allows us to conclude that

$$
\begin{aligned}
& \text { - } H_{(k)}^{q}\left(M, \omega_{t}\right) \cong \widetilde{H}_{\left[\omega_{t}^{k}\right]}^{q}(M) \text { for } k=1,3,4 \text { and for any } q \geq 6-k \text { and } t \in \mathbb{R} \\
& \text { - } H_{(2)}^{q}\left(M, \omega_{t}\right) \cong \widetilde{H}_{\left[\omega_{t}^{2}\right]}^{q}(M) \text { for any } q \geq 4 \text { if and only if } t \neq 0 .
\end{aligned}
$$

Therefore, for the compact symplectic manifold $\left(M, \omega_{0}\right)$ we have that the 2-coeffective cohomology is not isomorphic to the $\left[\omega^{2}\right]$-truncated de Rham cohomology. More precisely, one has that $H_{(2)}^{4}\left(M, \omega_{0}\right) \not \widetilde{H}_{\left[\omega_{0}^{2}\right]}^{4}(M)$.

\section{Flexibility of symplectic 2-step nilmanifolds}

In this section we find symplectic 2-step nilmanifolds of arbitrary high dimension which are $c$-flexible, $f$-flexible and $h$-flexible.

Proposition 8.1 Let $M=G / \Gamma$ be a 2-step nilmanifold of dimension $2 n \geq 6$, endowed with a continuous family of symplectic forms $\omega_{t}$. Then, the harmonic number $h_{3}\left(M, \omega_{t}\right)$ varies if and only if the coeffective number $\hat{c}_{3}\left(M, \omega_{t}\right)$ varies, if and only if the filtered number $\check{c}_{2 n-1}^{(n-2)}\left(M, \omega_{t}\right)$ varies. 
Proof Let us denote by $\mathfrak{g}$ the Lie algebra of the Lie group $G$. By [28, Theorem 3], for any symplectic 2-step nilmanifold

$$
b_{1}-h_{2 n-1}=\operatorname{dim}[\mathfrak{g}, \mathfrak{g}],
$$

which implies that the harmonic number $h_{2 n-1}$ does not depend on the symplectic form. From Theorem 4.4 we get $h_{3}-h_{2 n-1}=\hat{c}_{3}$. Therefore, $h_{3}$ varies if and only if $\hat{c}_{3}$ does.

On the other hand, by Definition 2.8 and Proposition 2.9 for $k=n-2$ we have

$$
-\hat{c}_{3}+\sum_{i=4}^{2 n}(-1)^{i} c_{i}^{(n-2)}=\chi^{(n-2)}=\sum_{r=3}^{2 n-2}(-1)^{r} b_{r} .
$$

Now, from Proposition 4.7 (ii) and Proposition 4.8 (i) for $k=n-2$, we get the following identities:

$$
c_{4}^{(n-2)}=\check{c}_{2 n-1}^{(n-2)}, \quad \text { and } \quad c_{i}^{(n-2)}=b_{i} \text { for every } \quad 5 \leq i \leq 2 n .
$$

Therefore, Eq. (21) reduces to

$$
-\hat{c}_{3}+\check{c}_{2 n-1}^{(n-2)}+\sum_{i=5}^{2 n-2}(-1)^{i} b_{i}-b_{2 n-1}+b_{2 n}=-b_{3}+b_{4}+\sum_{r=5}^{2 n-2}(-1)^{r} b_{r},
$$

which implies

$$
\check{c}_{2 n-1}^{(n-2)}=\hat{c}_{3}-b_{3}+b_{4}+b_{2 n-1}-b_{2 n} .
$$

Consequently, the filtered number $\check{c}_{2 n-1}^{(n-2)}\left(M, \omega_{t}\right)$ varies if and only if the coeffective number $\hat{c}_{3}\left(M, \omega_{t}\right)$ varies.

We apply Proposition 8.1 to a family of examples found by Sakane and Yamada [23]. The conclusion is that for any $k \geq 2$, there is a $6 k$-dimensional symplectic nilmanifold which is $c$-flexible, $f$-flexible and $h$-flexible.

Example 8.2 Let $k$ be an integer such that $k \geq 2$. We consider the $6 k$-dimensional compact nilmanifold $M=G / \Gamma$, where $G$ is a simply connected 2-step nilpotent Lie group defined by left-invariant 1 -forms $\left\{\alpha^{i}, \beta^{i}, 1 \leq i \leq 3 k\right\}$ satisfying

$$
\left\{\begin{array}{l}
d \alpha^{1}=\cdots=d \alpha^{3 k}=0 \\
d \beta^{1}=\alpha^{1} \wedge \alpha^{2} \\
d \beta^{2}=\alpha^{2} \wedge \alpha^{3} \\
\vdots \\
d \beta^{3 k-1}=\alpha^{3 k-1} \wedge \alpha^{3 k} \\
d \beta^{3 k}=\alpha^{3 k} \wedge \alpha^{1}
\end{array}\right.
$$

We denote by $\mathfrak{g}$ the Lie algebra of $G$. Let $\mathfrak{a}^{0}$ be a complementary vector subspace of the derived algebra $[\mathfrak{g}, \mathfrak{g}]$ in $\mathfrak{g}$, and consider $\mathfrak{a}^{1}=[\mathfrak{g}, \mathfrak{g}]$. So, $\mathfrak{g}=\mathfrak{a}^{0} \oplus \mathfrak{a}^{1}$ as a vector space, and this decomposition induces a bigraduation $\bigwedge^{r} \mathfrak{g}^{*}=\oplus_{i_{0}+i_{1}}=r \bigwedge^{i_{0}}\left(\mathfrak{a}^{0}\right)^{*} \otimes \bigwedge^{i_{1}}\left(\mathfrak{a}^{1}\right)^{*}$, for any $r$. For simplicity, we denote $\bigwedge^{i_{0}, i_{1}}=\bigwedge^{i_{0}}\left(\mathfrak{a}^{0}\right)^{*} \otimes \bigwedge^{i_{1}}\left(\mathfrak{a}^{1}\right)^{*}$. Hence, for $r=3$ we have

$$
\bigwedge^{3} \mathfrak{g}^{*}=\bigwedge^{3,0} \oplus \bigwedge^{2,1} \oplus \bigwedge^{1,2} \oplus \bigwedge^{0,3}
$$

Let $Z^{3}(\mathfrak{g})$ and $B^{3}(\mathfrak{g})$ denote the subspaces of $\bigwedge^{3} \mathfrak{g}^{*}$ consisting of closed and exact 3 -forms, respectively. 
For any invariant symplectic form $\omega$ on $M$, we denote by $\mathcal{H}^{3}(\mathfrak{g}, \omega)$ the space of invariant $\omega$-harmonic 3-forms. Since $M$ is 2-step, by [23, Theorem 3], we have that $B^{3}(\mathfrak{g}) \subset \mathcal{H}^{3}(\mathfrak{g}, \omega)$. Hence, the harmonic cohomology group $H_{\mathrm{hr}}^{3}(M, \omega)$ satisfies

$$
H_{\mathrm{hr}}^{3}(M, \omega) \cong \frac{\mathcal{H}^{3}(\mathfrak{g}, \omega)}{B^{3}(\mathfrak{g}) \cap \mathcal{H}^{3}(\mathfrak{g}, \omega)} \cong \frac{\mathcal{H}^{3}(\mathfrak{g}, \omega)}{B^{3}(\mathfrak{g})} .
$$

Moreover, it is easy to see that

$$
\mathcal{H}^{3}(\mathfrak{g}, \omega)=\bigwedge^{3,0} \oplus\left(Z^{3}(\mathfrak{g}) \cap \bigwedge^{2,1}\right) \oplus\left(\mathcal{H}^{3}(\mathfrak{g}, \omega) \cap \bigwedge^{1,2}\right),
$$

and

$$
B^{3}(\mathfrak{g}) \subset \bigwedge^{3,0} \oplus\left(Z^{3}(\mathfrak{g}) \cap \bigwedge^{2,1}\right)
$$

Hence, the harmonic number $h_{3}$ only depends on the dimension of the space $\mathcal{H}^{3}(\mathfrak{g}, \omega) \cap \bigwedge^{1,2}$.

Now, let us consider the symplectic form $\tau=a_{1} \alpha^{1} \wedge \beta^{1}+\cdots+a_{3 k} \alpha^{3 k} \wedge \beta^{3 k}$, where $a_{1}, \ldots, a_{3 k} \neq 0$. A direct calculation using (22) shows that

$$
\operatorname{dim}\left(\mathcal{H}^{3}(\mathfrak{g}, \tau) \cap \bigwedge^{1,2}\right)=0 .
$$

Let $\sigma=b_{1}\left(\alpha^{1} \wedge \beta^{2}-\alpha^{3} \wedge \beta^{1}\right)+\cdots+b_{3 k-2}\left(\alpha^{3 k-2} \wedge \beta^{3 k-1}-\alpha^{3 k} \wedge \beta^{3 k-2}\right)+b_{3 k-1}\left(\alpha^{3 k-1} \wedge\right.$ $\beta^{3 k}-\alpha^{1} \wedge \beta^{3 k-1}$ ). One can choose $b_{1}, \ldots, b_{3 k-1}$ such that $\sigma$ is non-degenerate (for more details see [23]) and, since it is closed, $\sigma$ defines another symplectic form on $M$. Using again the structure equations (22), one can prove that $\mathcal{H}^{3}(\mathfrak{g}, \sigma) \cap \wedge^{1,2}=\left\langle\alpha^{j+1} \wedge \beta^{j} \wedge \beta^{j+1} ; j=\right.$ $1, \ldots, 3 k\rangle$, which implies

$$
\operatorname{dim}\left(\mathcal{H}^{3}(\mathfrak{g}, \sigma) \cap \bigwedge^{1,2}\right)=3 k
$$

We take $\varepsilon>0$ sufficiently small so that the closed 2-form $\omega_{t}:=\sigma+t \tau$ is non-degenerate for any $t \in[0, \varepsilon]$. The space $\mathcal{H}^{3}\left(\mathfrak{g}, \omega_{t}\right) \cap \bigwedge^{1,2}$ has dimension $3 k$ for $t=0$, and dimension 0 for $t \neq 0$. This proves that the harmonic cohomology group $H_{\mathrm{hr}}^{3}\left(M, \omega_{t}\right)$ varies with $t$. Furthermore, since the harmonic number $h_{3}\left(M, \omega_{t}\right)$ varies, by Proposition 8.1, we get that the coeffective number $\hat{c}_{3}\left(M, \omega_{t}\right)$ and the filtered number $\breve{c}_{2 n-1}^{(n-2)}\left(M, \omega_{t}\right)$ also vary with $t$.

Acknowledgements This work has been partially supported by the Projects MTM2017-85649-P(AEI/FEDER, $\mathrm{UE}$ ), and E22-17R "Algebra y Geometría" (Gobierno de Aragón/FEDER). We thank the referee for useful suggestions and for comments on the relations of the generalized coeffective complex and the filtered complex of Tsai, Tseng and Yau.

\section{References}

1. Arnold, V.I., Khesin, B.A.: Topological Methods in Hydrodynamics. Applied Mathematical Sciences, vol. 125. Springer, Berlin (1998)

2. Angella, D., Kasuya, H.: Bott-Chern cohomology of solvmanifolds. Ann. Glob. Anal. Geom. 52, 363-411 (2017)

3. Angella, D., Tomassini, A.: Inequalities à la Frölicher and cohomological decompositions. J. Noncommut. Geom. 9, 505-542 (2015)

4. Baues, O.: Infra-solvmanifolds and rigidity of subgroups in solvable linear algebraic groups. Topology 43, 903-924 (2004)

5. Bock, C.: On low-dimensional solvmanifolds. Asian J. Math. 20, 199-262 (2016)

6. Bouché, T.: La cohomologie coeffective d'une variété symplectique. Bull. Sci. Math. 114(2), 115-122 (1990) 
7. Brylinski, J.-L.: A differential complex for Poisson manifolds. J. Differ. Geom. 28, 93-114 (1988)

8. Cho, Y.: Hard Lefschetz property of symplectic structures on compact Kähler manifolds. Trans. Am. Math. Soc. 368, 8223-8248 (2016)

9. Console, S., Fino, A.: On the de Rham cohomology of solvmanifolds. Ann. Sc. Norm. Super. Pisa Cl. Sci. (5) 10, 801-818 (2011)

10. Eastwood, M.: Extensions of the coeffective complex. Illinois J. Math. 57, 373-381 (2013)

11. Fernández, M., Ibáñez, R., de León, M.: A Nomizu's theorem for the coeffective cohomology. Math. Z. 226, 11-23 (1997)

12. Fernández, M., Ibáñez, R., de León, M.: Coeffective and de Rham cohomologies of symplectic manifolds. J. Geom. Phys. 27, 281-296 (1998)

13. Gompf, R.E.: A new construction of symplectic manifolds. Ann. Math. 142, 527-595 (1995)

14. Guan, Z.D.: Modification and the cohomology groups of compact solvmanifolds. Electron. Res. Announc. Am. Math. Soc. 13, 74-81 (2007)

15. Hattori, A.: Spectral sequence in the de Rham cohomology of fibre bundles. J. Fac. Sci. Univ. Tokyo 8(Sect. 1), 289-331 (1960)

16. Ibáñez, R., Rudyak, Y., Tralle, A., Ugarte, L.: On symplectically harmonic forms on six-dimensional nilmanifolds. Comment. Math. Helv. 76(1), 89-109 (2001). (Erratum 76 (2001), no 3, 576.)

17. Ibáñez, R., Rudyak, Yu., Tralle, A., Ugarte, L.: Symplectically harmonic cohomology of nilmanifolds. In: Symplectic and Contact Topology: Interactions and Perspectives (Toronto, ON/Montreal, QC, 2001), Fields Institute Communications, vol. 35, pp. 117-130. American Mathematical Society, Providence, RI (2003)

18. Kasuya, H.: Coeffective cohomology of symplectic aspherical manifolds. Proc. Am. Math. Soc. 140, 2835-2842 (2012)

19. Macrì, M.: Cohomological properties of unimodular six dimensional solvable Lie algebras. Differ. Geom. Appl. 31, 112-129 (2013)

20. Mathieu, O.: Harmonic cohomology classes of symplectic manifolds. Comment. Math. Helv. 70, 1-9 (1995)

21. Mostow, G.: Cohomology of topological groups and solvmanifolds. Ann. Math. 73, 20-48 (1961)

22. Nomizu, K.: On the cohomology of compact homogeneous space of nilpotent Lie group. Ann. Math. 59, 531-538 (1954)

23. Sakane, Y., Yamada, T.: Harmonic forms on compact symplectic 2-step nilmanifolds. In: Geometry, Integrability and Quantization (Sts. Constantine and Elena, 2002), pp. 257-270. Coral Press Scientific Publication, Sofia (2003)

24. Tanaka, H.L., Tseng, L.-S.: Odd sphere bundles, symplectic manifolds, and their intersection theory. Camb. J. Math. 6, 213-266 (2018)

25. Tsai, C.-J., Tseng, L.-S., Yau, S.-T.: Cohomology and Hodge theory on symplectic manifolds: III. J. Differ. Geom. 103, 83-143 (2016)

26. Tseng, L.-S., Yau, S.-T.: Cohomology and Hodge theory on symplectic manifolds: I. J. Differ. Geom. 91, 383-416 (2012)

27. Tseng, L.-S., Yau, S.-T.: Cohomology and Hodge theory on symplectic manifolds: II. J. Differ. Geom. 91, 417-444 (2012)

28. Yamada, T.: Harmonic cohomology groups of compact symplectic nilmanifolds. Osaka J. Math. 39, 363-381 (2002)

29. Yan, D.: Hodge structure on symplectic manifolds. Adv. Math. 120, 143-154 (1996)

Publisher's Note Springer Nature remains neutral with regard to jurisdictional claims in published maps and institutional affiliations. 